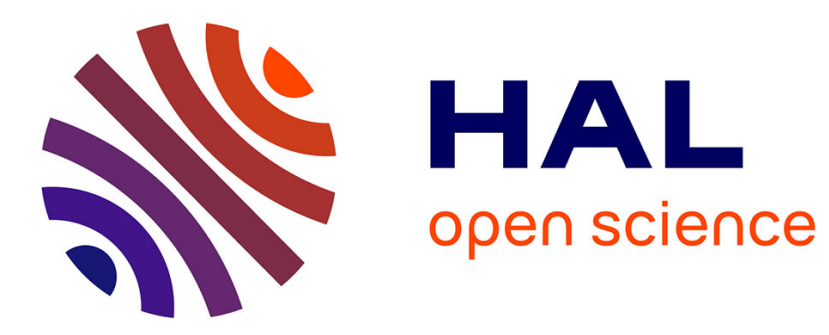

\title{
Lyapunov-Krasovskii functional for discretized homogeneous systems
}

Denis Efimov, Alexander y Aleksandrov

\section{To cite this version:}

Denis Efimov, Alexander y Aleksandrov. Lyapunov-Krasovskii functional for discretized homogeneous systems. SIAM Journal on Control and Optimization, 2021, 59 (4), pp.2546-2569. 10.1137/20M1340447. hal-03193489

\section{HAL Id: hal-03193489 \\ https://hal.inria.fr/hal-03193489}

Submitted on 8 Apr 2021

HAL is a multi-disciplinary open access archive for the deposit and dissemination of scientific research documents, whether they are published or not. The documents may come from teaching and research institutions in France or abroad, or from public or private research centers.
L'archive ouverte pluridisciplinaire HAL, est destinée au dépôt et à la diffusion de documents scientifiques de niveau recherche, publiés ou non, émanant des établissements d'enseignement et de recherche français ou étrangers, des laboratoires publics ou privés. 


\title{
Lyapunov-Krasovskii functional for discretized homogeneous systems
}

\author{
D. Efimov, A. Aleksandrov*
}

April 8, 2021

\begin{abstract}
The paper is devoted to stability analysis of discrete-time time-delay systems, obtained after explicit Euler discretization of (locally) homogeneous continuous-time models. The results are derived by applying the Lyapunov-Krasovskii theory. A generic structure of the functional is given that suits for any homogeneous system of non-zero degree (and it can also be used for any dynamics admitting a homogeneous approximation). The obtained conditions are utilized to demonstrate stability for a discretized delayed locally homogeneous planar system with negative and positive degrees.
\end{abstract}

\section{Introduction}

Presence of delays in the modern interconnected plants is hard to neglect, especially for networked and distributed large-scale systems. Delays and lags may influence the system stability, as well as quality of transients, see $[1,2,3]$. Synthesis of control and estimation algorithms, which take into account uncertain or time-varying delays, is a popular subject of research $[4,5,6]$. For stability analysis of time-delay systems, a LyapunovKrasovskii functional or a Lyapunov-Razumikhin function can be used [7], and there are plenty of results in the domain, e.g., [8, 9], mainly related with linear dynamics [10]. The popularity of the latter is based on a wide choice of available Lyapunov-Krasovskii functionals and Lyapunov-Razumikhin functions, which is also the case for some classes of nonlinear plants, as port-Hamiltonian systems [11], for instance.

Another promising class of canonical nonlinear dynamical models, which disposes a variety of tools, is composed by homogeneous systems that take an intermediate place between linear and nonlinear ones [12]. The theory of homogeneous dynamical systems has been established, first, for ordinary differential equations $[13,14,15,16]$, and next extended to differential inclusions $[17,18,12]$, time-delay systems [19, 20], discrete-time systems [21] and partial differential equations [22]. The main feature of a homogeneous nonlinear model is that its local behavior is the same as the global one, the homogeneous stable/unstable systems admit homogeneous Lyapunov/Chetaev functions $[16,23,24,25,26,27]$ and possess robustness properties with respect to external inputs and delays $[28,29,20,30]$. Using the concept of local homogeneity [16, 31, 25, 32] a generic dynamical system may be locally approximated by a homogeneous one, which allows the set of methods developed for this class of dynamics to be applied at large.

It has been shown that homogeneous systems have certain robustness of stability with respect to delays $[33,34,35,36,37,20,30]$. In particular, for the case of positive degree it has been proven that homogeneity and global asymptotic stability in the delay-free case imply local asymptotic stability for any value of delay. For the case of negative degree, under similar restrictions, for any delay, global asymptotic stability with respect to a compact set containing the origin is obtained. The latter results have been obtained in $[20,30]$ using Lyapunov-Razumikhin theory, and in [38, 39] by applying Lyapunov-Krasovskii approach.

In the present work, following the results of $[40,38,39]$, we consider a discrete-time system obtained after discretization (using the explicit Euler method) of a (locally) homogeneous system with non-zero degree, which is asymptotically stable in the delay-free case. The stability conditions are derived by proposing a generic structure of Lyapunov-Krasovskii functional for this class of dynamics. The presented approach allows the convergence rates and the domain of stability/attraction to be evaluated. It is demonstrated that for a homogeneous timedelay system (asymptotically stable for zero delay) the Euler discretization preserves the rates of decay, which is not the case for non-delayed homogeneous dynamics [41].

The outline of this paper is as follows. The preliminary definitions and the homogeneity for delay-free systems are given in Section 2. The problem statement and the main results are presented in Section 3, their

${ }^{*}$ D. Efimov is with Univ. Lille, Inria, CNRS, UMR 9189 - CRIStAL, F-59000 Lille, France and ITMO University, 49 av. Kronverkskiy, 197101 Saint Petersburg, Russia. A. Aleksandrov is with Saint Petersburg State University, 7-9 Universitetskaya nab., 199034 Saint Petersburg, Russia. This work was partially supported by the Ministry of Science and Higher Education of Russian Federation, passport of goszadanie no. 2019-0898, and by the Russian Foundation for Basic Research (grant no. 19-0100146-a). 
extension to locally homogeneous systems is developed in Section 4. An illustrative example is considered in Section 5 .

\section{Preliminaries}

In the sequel, $\mathbb{R}$ denotes the field of real numbers, and $\mathbb{R}^{n}$ is the $n$-dimensional Euclidean space; $\mathbb{R}_{+}=\{s \in \mathbb{R}$ : $s \geq 0\}$. The Euclidean norm $|x|$ will be used for vectors $x \in \mathbb{R}^{n}$.

A continuous function $\sigma: \mathbb{R}_{+} \rightarrow \mathbb{R}_{+}$belongs to class $\mathcal{K}$ if it is strictly increasing and $\sigma(0)=0$; it belongs to class $\mathcal{K}_{\infty}$ if it is also unbounded.

\subsection{Convergence conditions}

We will need the following results estimating the rate of convergence for discrete-time scalar nonlinear systems:

Lemma 1. [40] If a sequence $\left\{v_{k}\right\}_{k=0}^{+\infty}$ satisfies the condition

$$
0 \leq v_{k+1} \leq v_{k}-\alpha v_{k}^{1+\varrho}
$$

with $\alpha>0, \varrho>0, v_{0} \in\left[0, \frac{\alpha^{-\varrho^{-1}}}{(1+\varrho)^{-1}}\right]$, then

for $k=0,1, \ldots$

$$
v_{k} \leq \frac{v_{0}}{\left(1+\alpha \varrho v_{0}^{\varrho} k\right)^{-1}}
$$

In a similar way, it can be proven that the following lemma is valid:

Lemma 2. If a sequence $\left\{v_{k}\right\}_{k=0}^{N}$ with $N>0$ satisfies the condition

$$
0 \leq v_{k+1} \leq v_{k}-\alpha v_{k}^{1-\varrho},
$$

for $k=0,1, \ldots, N$, where $\alpha>0, \varrho \in(0,1), v_{0} \geq \alpha^{\varrho^{-1}}$, then

$$
v_{k} \leq v_{0}\left(1-\alpha \varrho v_{0}^{-\varrho} k\right)^{\varrho^{-1}}
$$

for $k=0,1, \ldots, N$ with $N \leq N_{0}=\frac{v_{0}^{\varrho}-\alpha}{\alpha \varrho}$.

Proof. Let us use the same arguments as in the proof of Lemma 1 [40].

Note that the restriction $v_{k+1} \geq 0$ follows $v_{k} \geq \alpha^{\varrho^{-1}}$ for all $k=0,1, \ldots, N$, then the upper estimate verifies this condition for $\frac{v_{0}^{\varrho}-\alpha}{\alpha \varrho} \geq k$.

By induction, the estimate obviously holds for $k=0$, and assuming that it is satisfied for all $k \leq l$ for some integer $l \in\left[1, N_{0}-1\right)$ let us demonstrate that it is valid in such a case for $k=l+1$. Hence,

$$
\begin{gathered}
v_{l+1} \leq v_{l}\left(1-\alpha v_{l}^{-\varrho}\right) \leq v_{0}\left(1-\alpha \varrho v_{0}^{-\varrho} l\right)^{\varrho^{-1}}\left(1-\frac{\alpha v_{0}^{-\varrho}}{1-\alpha \varrho v_{0}^{-\varrho} l}\right) \\
=v_{0}\left(1-\alpha \varrho v_{0}^{-\varrho}(l+1)\right)^{\varrho^{-1}} g(z, l),
\end{gathered}
$$

where $z=\alpha \varrho v_{0}^{-\varrho}$ and

$$
g(z, l)=\left(\frac{1-z l}{1-z(l+1)}\right)^{\varrho^{-1}}\left(1-\frac{1}{\varrho} \frac{z}{1-z l}\right) .
$$

It is necessary to establish the conditions providing $g(z, l) \leq 1$ for any $z>0$ and $l \in\left[1, N_{0}-1\right)$. Note that the restriction on $l$ and $N_{0}$ implies that $(\alpha \varrho(l+1))^{\varrho^{-1}}<v_{0}$, and we have $1-z(l+1)>0$ for any $l \in\left[1, N_{0}-1\right)$ (thus, $1-z l>0$ also). For $g(z, 0)=\left(\frac{1}{1-z}\right)^{\varrho^{-1}}\left(1-\frac{z}{\varrho}\right)$ we get that $g(z, 0) \leq 1$ (indeed, $g(0,0)=1$ and $\frac{\partial g(z, 0)}{\partial z}=\frac{z}{\varrho^{2}} \frac{\varrho-1}{(1-z)^{\frac{1+\varrho}{\varrho}}}<0$ for $z \in(0,1)$, which is the case for $\left.v_{0}>\alpha^{\varrho^{-1}}>(\varrho \alpha)^{\varrho^{-1}}\right)$, then

$$
\frac{\partial g(z, l)}{\partial l}=-\frac{z^{3}(1-\varrho)}{\varrho^{2}(1-z l)^{2}(1-z(l+1))}\left(\frac{1-z l}{1-z(l+1)}\right)^{\varrho^{-1}}
$$

and it is always negative for $l \in\left[1, N_{0}-1\right)$, which implies the desired result.

In the conditions of this lemma, we have that from any initial value $v_{0}$ satisfying the introduced restrictions, the corresponding sequence converges to the zone

$$
v_{k} \leq \alpha^{\varrho^{-1}}
$$

for $k \leq N_{0}$, and it stays there if additionally $0 \leq v_{k+1} \leq v_{k}$ for all $k=0,1, \ldots$ 


\subsection{Homogeneity}

For any $r_{i}>0, i=\overline{1, n}$ and $\lambda>0$, define the dilation matrix $\Lambda_{r}(\lambda)=\operatorname{diag}\left\{\lambda^{r_{i}}\right\}_{i=1}^{n}$ and the vector of weights $\mathbf{r}=\left[r_{1}, \ldots, r_{n}\right]^{\top}, r_{\max }=\max _{1 \leq j \leq n} r_{j}$ and $r_{\min }=\min _{1 \leq j \leq n} r_{j}$.

For any $\mathbf{r}$ and $x \in \mathbb{R}^{n}$ the homogeneous norm can be defined, for example, as follows

$$
|x|_{r}=\left(\sum_{i=1}^{n}\left|x_{i}\right|^{\vartheta / r_{i}}\right)^{1 / \vartheta}, \vartheta \geq r_{\max } .
$$

For all $x \in \mathbb{R}^{n}$, its Euclidean norm $|x|$ is related with the homogeneous one:

$$
\underline{\sigma}_{r}\left(|x|_{r}\right) \leq|x| \leq \bar{\sigma}_{r}\left(|x|_{r}\right)
$$

for some $\underline{\sigma}_{r}, \bar{\sigma}_{r} \in \mathcal{K}_{\infty}[27]$. The homogeneous norm has an important property that is $\left|\Lambda_{r}(\lambda) x\right|_{r}=\lambda|x|_{r}$ for all $x \in \mathbb{R}^{n}$.

Define $\mathbb{S}_{r}=\left\{x \in \mathbb{R}^{n}:|x|_{r}=1\right\}$ and $\mathbb{B}_{r}=\left\{x \in \mathbb{R}^{n}:|x|_{r} \leq 1\right\}$ as the unit sphere and the unit ball in the homogeneous norm, respectively.

Definition 1. [16, 13] The function $g: \mathbb{R}^{n} \rightarrow \mathbb{R}$ is called $\mathbf{r}$-homogeneous if for any $x \in \mathbb{R}^{n}$ the relation

$$
g\left(\Lambda_{r}(\lambda) x\right)=\lambda^{d} g(x)
$$

holds for some $d \in \mathbb{R}$ and all $\lambda>0$.

The vector field $f: \mathbb{R}^{n} \rightarrow \mathbb{R}^{n}$ is called $\mathbf{r}$-homogeneous if for any $x \in \mathbb{R}^{n}$ the relation

$$
f\left(\Lambda_{r}(\lambda) x\right)=\lambda^{d} \Lambda_{r}(\lambda) f(x)
$$

holds for some $d \geq-r_{\min }$ and all $\lambda>0$.

In both cases, the constant $d$ is called the degree of homogeneity.

A system $\dot{x}=f(x)$ is called homogeneous if $f$ is $\mathbf{r}$-homogeneous. An advantage of homogeneous systems is that any its solution can be obtained from another solution under the dilation and a suitable time reparametrization, and that homogeneous asymptotically stable system admits a homogeneous Lyapunov function [16, 13]. If $f(x)=A x$ for some $A \in \mathbb{R}^{n \times n}$, then it is $\mathbf{r}$-homogeneous of zero degree for $\mathbf{r}=[1, \ldots, 1]^{\top}$, and the properties of scalability of solutions and existence of a homogeneous (quadratic) Lyapunov function are well-known for linear systems, then homogeneity allows these useful features to be extended on a wider class of dynamics.

Lemma 3. Let $h: \mathbb{R}^{n} \rightarrow \mathbb{R}$ be $\chi$-Hölder continuous on $\mathbb{B}_{r}$ with constant $L>0$ and $\mathbf{r}$-homogeneous function of degree $\nu \geq \chi r_{\min }$, then

$$
|h(x)-h(z)| \leq L \max \left\{1,|x|_{r}^{\nu-\chi r_{\min }},|z|_{r}^{\nu-\chi r_{\min }}\right\}|x-z|^{\chi}
$$

for all $x, z \in \mathbb{R}^{n}$.

Proof. By definition of homogeneity $h(\Lambda(\lambda) x)=\lambda^{\nu} h(x)$ for any $x \in \mathbb{R}^{n}$ and $\lambda>0$. Take any $x, z \in \mathbb{R}^{n}$ and introduce $\lambda=\max \left\{|x|_{r},|z|_{r}\right\}$. If $\lambda \leq 1$, then

$$
|h(x)-h(z)| \leq L|x-z|^{\chi}
$$

by the properties of $L$, and the required estimate holds. Let $\lambda>1$, then there exist $\xi, \zeta \in \mathbb{B}_{r}$ such that $x=\Lambda(\lambda) \xi$ and $z=\Lambda(\lambda) \zeta$, hence

$$
\begin{gathered}
|h(x)-h(z)|=|h(\Lambda(\lambda) \xi)-h(\Lambda(\lambda) \zeta)|=\lambda^{\nu}|h(\xi)-h(\zeta)| \\
\leq L|\xi-\zeta|^{\chi} \lambda^{\nu}=L\left|\Lambda^{-1}(\lambda) x-\Lambda^{-1}(\lambda) z\right|^{\chi} \lambda^{\nu} \leq L\left|\Lambda^{-1}(\lambda)\right|^{\chi}|x-z|^{\chi} \lambda^{\nu},
\end{gathered}
$$

and recalling that

$$
\left|\Lambda^{-1}(\lambda)\right| \leq \begin{cases}\lambda^{-r_{\min }} & \lambda>1 \\ \lambda^{-r_{\max }} & \lambda \leq 1\end{cases}
$$

we obtain the desired result.

This lemma shows that if a homogeneous function is Lipschitz or Hölder continuous in $\mathbb{B}_{r}$ with a constant $L$, then it possesses this property on any compact set $\mathcal{X} \subset \mathbb{R}^{n}$ with the constant

$$
L \max \left\{1, \sup _{x \in \mathcal{X}}|x|_{r}^{\nu-\chi r_{\min }}\right\} .
$$




\subsection{Useful inequalities}

Finally, Young's inequality claims that for any $\mathfrak{a}, \mathfrak{b} \in \mathbb{R}_{+}$and $\gamma>0, \delta>0$ :

$$
\mathfrak{a}^{\gamma} \mathfrak{b}^{\delta} \leq \frac{1}{p} \mathfrak{a}^{\gamma p}+\frac{p-1}{p} \mathfrak{b}^{\frac{\delta p}{p-1}}
$$

for any $p>1$. Jensen's inequality for any convex $\kappa_{\cup}: \mathbb{R} \rightarrow \mathbb{R}$ or concave $\kappa_{\cap}: \mathbb{R} \rightarrow \mathbb{R}$ function reads:

$$
\kappa_{\cup}\left(\frac{\sum_{i=1}^{n} x_{i}}{n}\right) \leq \frac{\sum_{i=1}^{n} \kappa_{\cup}\left(x_{i}\right)}{n}, \kappa_{\cap}\left(\frac{\sum_{i=1}^{n} x_{i}}{n}\right) \geq \frac{\sum_{i=1}^{n} \kappa_{\cap}\left(x_{i}\right)}{n}
$$

for any $x_{1}, \ldots, x_{n} \in \mathbb{R}$. In addition,

$$
\left(\sum_{i=1}^{n} x_{i}\right)^{\gamma} \leq \sum_{i=1}^{n} x_{i}^{\gamma}
$$

for any $x_{1}, \ldots, x_{n} \in \mathbb{R}_{+}$and $\gamma \in[0,1)$.

Another related result can be obtained using the properties of homogeneous functions and a specially defined homogeneous norm:

Lemma 4. [39] Let $\mathfrak{a}, \mathfrak{b} \in \mathbb{R}_{+}$and $\ell>0, \alpha>0, \beta>0, \gamma>0, \delta>0$ be given, then

$$
\mathfrak{a}^{\alpha}+\mathfrak{b}^{\beta}-\ell \mathfrak{a}^{\gamma} \mathfrak{b}^{\delta} \geq 0
$$

provided that

1) $\max \left\{\mathfrak{a}^{\alpha}, \mathfrak{b}^{\beta}\right\} \geq \ell^{\frac{1}{1-\frac{\gamma}{\alpha}-\frac{\delta}{\beta}}}$ and $\frac{\gamma}{\alpha}+\frac{\delta}{\beta}<1$,

2) $\max \left\{\mathfrak{a}^{\alpha}, \mathfrak{b}^{\beta}\right\} \leq \ell^{\frac{1}{1-\frac{\gamma}{\alpha}-\frac{\delta}{\beta}}}$ and $\frac{\gamma}{\alpha}+\frac{\delta}{\beta}>1$.

\section{$3 \quad$ Main Results}

In this section, first, the system of interest will be introduced with the basic hypotheses. Next, two cases will be analyzed with positive and negative homogeneous degrees.

\subsection{Problem statement}

Consider the difference system

$$
x(k+1)=x(k)+h F(x(k), x(k-m)),
$$

where $x(k) \in \mathbb{R}^{n}$ and $x^{(k)}=\left(x^{T}(k), x^{T}(k-1), \ldots, x^{T}(k-m)\right)^{T} \in \mathbb{R}^{n(m+1)}$ is the augmented state vector, $m$ is a nonnegative integer representing delay; $h>0$ is a discretization step, $k=0,1, \ldots$ is the discrete time; the vector function $F: \mathbb{R}^{2 n} \rightarrow \mathbb{R}^{n}$ is locally bounded in $\mathbb{R}^{2 n}$. Denote by $x\left(k, x^{(0)}\right)$ a solution of (1) with initial conditions $x^{(0)} \in \mathbb{R}^{n(m+1)}$, and $x^{(k)}\left(x^{(0)}\right)$ as the corresponding state trajectory.

The system (1) is a discrete-time counterpart of the continuous-time system

$$
\dot{z}(t)=F(z(t), z(t-m h))
$$

obtained by applying the explicit Euler discretization method with the step $h$.

Assumption 1. The vector field $F$ is $\mathbf{r}$-homogeneous of degree $\nu \in\left(-r_{\min }, 0\right) \cup(0,+\infty)$ :

$$
F(\Lambda(\lambda) \xi, \Lambda(\lambda) \zeta)=\lambda^{\nu} \Lambda(\lambda) F(\xi, \zeta) \quad \forall \xi, \zeta \in \mathbb{R}^{n}, \forall \lambda>0
$$

and uniformly continuous with respect to the first argument in $\mathbb{B}_{r}$ : for any $L>0$ there exists $\chi>0$ such that

$$
|F(\phi+\varphi, \psi)-F(\phi, \psi)| \leq L
$$

for any $\phi, \psi \in \mathbb{B}_{r}$ with $|\varphi| \leq \chi$.

This continuity hypothesis is satisfied if, for example, $F$ is continuous in $\mathbb{R}^{2 n}$. We will need also stability of the corresponding delay-free system:

Assumption 2. The zero solution of

$$
\dot{z}(t)=F(z(t), z(t))
$$

is asymptotically stable. 
Following [16, 13], let $V(z)$ be a twice continuously differentiable $\mathbf{r}$-homogeneous Lyapunov function of the degree $\mu>r_{\max }$ constructed for the delay-free (homogeneous) system from Assumption 2 and satisfying the conditions of the Lyapunov asymptotic stability theorem [13].

Hence, it is assumed that a dynamical system (2) is given, which is asymptotically stable and homogeneous with non-zero degree for the delay-free case in continuous time (assumptions 1 and 2). Under these restrictions and for any $m h \geq 0$, the origin is asymptotically stable for (2) with $\nu>0$ and there is a vicinity of the origin that globally attracts all trajectories of $(2)$ with $\nu<0[19,30]$. Let us consider its discretization (1) obtained by the explicit Euler method, and the objective is to determine the conditions and the kind of the discrete-time system (1) stability under introduced hypotheses. To this end, the Lyapunov-Krasovskii theory will be used.

Note that the case of $\nu=0$ is excluded by Assumption 1, hence, in the sequel the case of linear dynamics in (1), which is well studied already [10], is not addressed. Recall that in the delay-free continuous-time setting, an asymptotically stable homogeneous system has a faster converge rate than any linear dynamics close to the origin (finite-time stability) for a negative degree and at infinity (nearly fixed-time stability) for a positive degree [12].

\subsection{Lyapunov-Krasovskii functional}

Choose a Lyapunov-Krasovskii functional candidate for the system (1) as follows

$$
\begin{aligned}
W\left(x^{(k)}\right)= & V(x(k))+h\left(\frac{\partial V(x(k))}{\partial z}\right)^{T} \sum_{i=1}^{m} F(x(k), x(k-i)) \\
& +h \sum_{i=1}^{m} \beta_{i}|x(k-i)|_{r}^{\rho}
\end{aligned}
$$

where $\rho, \beta_{1}, \ldots, \beta_{m}$ are positive parameters, whose values will be defined later. It is a functional of the complete type [42], which is based on the Lyapunov function $V$ of the delay-free continuous-time counterpart of (1), and its feature consists in the introduction of a sign-indefinite middle term, whose advantages can be effectively utilized due to the homogeneity [39]. We will use the following norm in the sequel:

$$
\left\|x^{(k)}\right\|=\max \left\{|x(k)|_{r},|x(k-1)|_{r}, \ldots,|x(k-m)|_{r}\right\} .
$$

Using the homogeneity of $V$ and $F$ we obtain:

$$
\begin{gathered}
h \sum_{i=1}^{m} \beta_{i}|x(k-i)|_{r}^{\rho}-a_{3} h \sum_{j=1}^{n}|x(k)|_{r}^{\mu-r_{j}} \sum_{i=1}^{m}\left(|x(k)|_{r}^{\nu+r_{j}}+|x(k-i)|_{r}^{\nu+r_{j}}\right) \\
+a_{1}|x(k)|_{r}^{\mu} \leq W\left(x^{(k)}\right) \leq a_{2}|x(k)|_{r}^{\mu}+h \sum_{i=1}^{m} \beta_{i}|x(k-i)|_{r}^{\rho} \\
+a_{3} h \sum_{j=1}^{n}|x(k)|_{r}^{\mu-r_{j}} \sum_{i=1}^{m}\left(|x(k)|_{r}^{\nu+r_{j}}+|x(k-i)|_{r}^{\nu+r_{j}}\right),
\end{gathered}
$$

where $a_{1}, a_{2}, a_{3}$ are the positive constants, which may be evaluated as follows:

$$
\begin{gathered}
a_{1}=\inf _{y \in \mathbb{S}_{r}} V(y), a_{2}=\sup _{y \in \mathbb{S}_{r}} V(y), \\
a_{3}=\sup _{\phi, \psi \in \mathbb{B}_{r}}\left|\left(\frac{\partial V(\phi)}{\partial \phi}\right)^{T} F(\phi, \psi)\right| .
\end{gathered}
$$

Consider the difference $\Delta W=W\left(x^{(k+1)}\right)-W\left(x^{(k)}\right)$ of $W$ along the solutions of (1):

$$
\begin{gathered}
\Delta W=h\left(\frac{\partial V\left(x(k)+h \theta_{1 k} F(x(k), x(k-m))\right)}{\partial z}\right)^{T} F(x(k), x(k-m)) \\
+h \sum_{i=1}^{m} \beta_{i}|x(k-i+1)|_{r}^{\rho}-h \sum_{i=1}^{m} \beta_{i}|x(k-i)|_{r}^{\rho} \\
+h\left(\frac{\partial V(x(k)+h F(x(k), x(k-m)))}{\partial z}\right)^{T} \sum_{i=1}^{m} F(x(k+1), x(k-i+1)) \\
-h\left(\frac{\partial V(x(k))}{\partial z}\right)^{T} \sum_{i=1}^{m} F(x(k), x(k-i)),
\end{gathered}
$$


where $\theta_{1 k} \in(0,1)$ comes from application of the Mean Value Theorem to the difference $V(x(k+1))-V(x(k))$. Further,

$$
\begin{gathered}
\Delta W=h\left(\frac{\partial V(x(k))}{\partial z}\right)^{T} F(x(k), x(k-m)) \\
+h\left(\frac{\partial V\left(x(k)+h \theta_{1 k} F(x(k), x(k-m))\right)}{\partial z}-\frac{\partial V(x(k))}{\partial z}\right)^{T} F(x(k), x(k-m)) \\
+h \beta_{1}|x(k)|_{r}^{\rho}-h \beta_{m}|x(k-m)|_{r}^{\rho}+h \sum_{j=1}^{m-1}\left(\beta_{j+1}-\beta_{j}\right)|x(k-j)|_{r}^{\rho} \\
+h\left(\frac{\partial V(x(k)+h F(x(k), x(k-m)))}{\partial z}-\frac{\partial V(x(k))}{\partial z}\right)^{T} \sum_{i=1}^{m} F(x(k+1), x(k-i+1)) \\
+h\left(\frac{\partial V(x(k))}{\partial z}\right)^{T}\left(\sum_{i=1}^{m} F(x(k+1), x(k-i+1))-\sum_{i=1}^{m} F(x(k), x(k-i+1))\right) \\
+h\left(\frac{\partial V(x(k))}{\partial z}\right)^{T}\left(\sum_{i=1}^{m} F(x(k), x(k-i+1))-\sum_{i=1}^{m} F(x(k), x(k-i))\right)
\end{gathered}
$$

and making a direct sum of the first and the last terms we obtain:

$$
\begin{gathered}
\Delta W=h\left(\frac{\partial V(x(k))}{\partial z}\right)^{T} F(x(k), x(k)) \\
+h \beta_{1}|x(k)|_{r}^{\rho}-h \beta_{m}|x(k-m)|_{r}^{\rho}+h \sum_{j=1}^{m-1}\left(\beta_{j+1}-\beta_{j}\right)|x(k-j)|_{r}^{\rho} \\
+h\left(\frac{\partial V\left(x(k)+h \theta_{1 k} F(x(k), x(k-m))\right)}{\partial z}-\frac{\partial V(x(k))}{\partial z}\right)^{T} F(x(k), x(k-m)) \\
+h\left(\frac{\partial V(x(k)+h F(x(k), x(k-m)))}{\partial z}-\frac{\partial V(x(k))}{\partial z}\right)^{T} \sum_{i=1}^{m} F(x(k+1), x(k-i+1)) \\
+h\left(\frac{\partial V(x(k))}{\partial z}\right)^{T}\left(\sum_{i=1}^{m} F(x(k+1), x(k-i+1))-\sum_{i=1}^{m} F(x(k), x(k-i+1))\right)
\end{gathered}
$$

Hence, due to the homogeneity of $V$ and $F$ the following estimate can be substantiated:

$$
\begin{gathered}
\Delta W \leq-a_{4} h|x(k)|_{r}^{\mu+\nu}+h \beta_{1}|x(k)|_{r}^{\rho}-h \beta_{m}|x(k-m)|_{r}^{\rho}+h \sum_{j=1}^{m-1}\left(\beta_{j+1}-\beta_{j}\right)|x(k-j)|_{r}^{\rho} \\
+h a_{5} \sum_{l=1}^{n}\left|\frac{\partial V\left(x(k)+h \theta_{1 k} F(x(k), x(k-m))\right)}{\partial z_{l}}-\frac{\partial V(x(k))}{\partial z_{l}}\right|\left(|x(k)|_{r}^{\nu+r_{l}}+|x(k-m)|_{r}^{\nu+r_{l}}\right) \\
+h a_{5} \sum_{l=1}^{n}\left|\frac{\partial V(x(k)+h F(x(k), x(k-m)))}{\partial z_{l}}-\frac{\partial V(x(k))}{\partial z_{l}}\right| \sum_{i=1}^{m}\left(|x(k+1)|_{r}^{\nu+r_{l}}+|x(k-i+1)|_{r}^{\nu+r_{l}}\right) \\
+h a_{6} \sum_{l=1}^{n}|x(k)|_{r}^{\mu-r_{l}} \sum_{i=1}^{m}\left|F_{l}(x(k+1), x(k-i+1))-F_{l}(x(k), x(k-i+1))\right|
\end{gathered}
$$

where $a_{4}, a_{5}, a_{6}$ are positive constants, which may be derived as

$$
\begin{gathered}
a_{4}=-\sup _{y \in \mathbb{S}_{r}}\left(\frac{\partial V(y)}{\partial y}\right)^{T} F(y, y), a_{5}=\sup _{\phi, \psi \in \mathbb{B}_{r}}|F(\phi, \psi)|, \\
a_{6}=\sup _{y \in \mathbb{S}_{r}}\left|\frac{\partial V(y)}{\partial y}\right| .
\end{gathered}
$$

Note that in such a case $a_{3} \leq a_{5} a_{6}$. 
For brevity of notation denote $\lambda=\left\|x^{(k)}\right\|$ and recall that $\Lambda(\lambda)=\operatorname{diag}\left\{\lambda^{r_{1}}, \ldots, \lambda^{r_{n}}\right\}$ (we assume that $\lambda \neq 0$ ). Then, applying again the Mean Value Theorem we obtain:

$$
\begin{gathered}
\left|\frac{\partial V\left(x(k)+h \theta_{1 k} F(x(k), x(k-m))\right)}{\partial z_{l}}-\frac{\partial V(x(k))}{\partial z_{l}}\right|\left(|x(k)|_{r}^{\nu+r_{l}}+|x(k-m)|_{r}^{\nu+r_{l}}\right) \\
\leq a_{5} h \sum_{s=1}^{n}\left|\frac{\partial^{2} V\left(x(k)+h \theta_{2 k l} F(x(k), x(k-m))\right)}{\partial z_{l} \partial z_{s}}\right| \\
\times\left(|x(k)|_{r}^{\nu+r_{s}}+|x(k-m)|_{r}^{\nu+r_{s}}\right)\left(|x(k)|_{r}^{\nu+r_{l}}+|x(k-m)|_{r}^{\nu+r_{l}}\right) \\
=a_{5} h \sum_{s=1}^{n} \lambda^{\mu-r_{l}-r_{s}}\left|\frac{\partial^{2} V\left(\Lambda^{-1}(\lambda) x(k)+h \theta_{2 k l} \lambda^{\nu} F\left(\Lambda^{-1}(\lambda) x(k), \Lambda^{-1}(\lambda) x(k-m)\right)\right)}{\partial z_{l} \partial z_{s}}\right| \\
\times\left(|x(k)|_{r}^{\nu+r_{s}}+|x(k-m)|_{r}^{\nu+r_{s}}\right)\left(|x(k)|_{r}^{\nu+r_{l}}+|x(k-m)|_{r}^{\nu+r_{l}}\right) \\
\leq 4 a_{5} h \lambda^{2 \nu+\mu} \sum_{s=1}^{n}\left|\frac{\partial^{2} V\left(\Lambda^{-1}(\lambda) x(k)+h \theta_{2 k l} \lambda^{\nu} F\left(\Lambda^{-1}(\lambda) x(k), \Lambda^{-1}(\lambda) x(k-m)\right)\right)}{\partial z_{l} \partial z_{s}}\right| \\
\leq 4 a_{5} h \sum_{p=0}^{m}|x(k-p)|_{r}^{2 \nu+\mu} \sum_{s=1}^{n}\left|\frac{\partial^{2} V\left(\Lambda^{-1}(\lambda) x(k)+h \theta_{2 k l} \lambda^{\nu} F\left(\Lambda^{-1}(\lambda) x(k), \Lambda^{-1}(\lambda) x(k-m)\right)\right)}{\partial z_{l} \partial z_{s}}\right|,
\end{gathered}
$$

where $\theta_{2 k l} \in(0,1)$.

In a similar way, we obtain for $\theta_{3 k l} \in(0,1)$ :

$$
\begin{gathered}
\left|\frac{\partial V(x(k)+h F(x(k), x(k-m)))}{\partial z_{l}}-\frac{\partial V(x(k))}{\partial z_{l}}\right| \sum_{i=1}^{m}\left(|x(k+1)|_{r}^{\nu+r_{l}}+|x(k-i+1)|_{r}^{\nu+r_{l}}\right) \\
\leq 2 a_{5} h \lambda^{\nu+\mu-r_{l}} \sum_{s=1}^{n}\left|\frac{\partial^{2} V\left(\Lambda^{-1}(\lambda) x(k)+h \theta_{3 k l} \lambda^{\nu} F\left(\Lambda^{-1}(\lambda) x(k), \Lambda^{-1}(\lambda) x(k-m)\right)\right)}{\partial z_{l} \partial z_{s}}\right| \\
\times\left(m \lambda^{\nu+r_{l}}\left|\Lambda^{-1}(\lambda) x(k)+h \lambda^{\nu} F\left(\Lambda^{-1}(\lambda) x(k), \Lambda^{-1}(\lambda) x(k-m)\right)\right|^{\nu+r_{l}}+\sum_{i=1}^{m}|x(k-i+1)|_{r}^{\nu+r_{l}}\right) \\
\leq 2 m a_{5} h \sum_{p=0}^{m}|x(k-p)|_{r}^{2 \nu+\mu} \sum_{s=1}^{n}\left|\frac{\partial^{2} V\left(\Lambda^{-1}(\lambda) x(k)+h \theta_{3 k l} \lambda^{\nu} F\left(\Lambda^{-1}(\lambda) x(k), \Lambda^{-1}(\lambda) x(k-m)\right)\right)}{\partial z_{l} \partial z_{s}}\right| \\
\times\left(\left|\Lambda^{-1}(\lambda) x(k)+h \lambda^{\nu} F\left(\Lambda^{-1}(\lambda) x(k), \Lambda^{-1}(\lambda) x(k-m)\right)\right|^{\nu+r_{l}}+1\right) .
\end{gathered}
$$

Define $\omega=\max \left\{|x(k+1)|_{r}, \lambda\right\}$, recall that $\lambda \neq 0$ and that due to imposed restrictions on $F$ we get

$$
\begin{gathered}
\sum_{l=1}^{n}|x(k)|_{r}^{\mu-r_{l}} \sum_{i=1}^{m}\left|F_{l}(x(k+1), x(k-i+1))-F_{l}(x(k), x(k-i+1))\right| \\
=\sum_{l=1}^{n}|x(k)|_{r}^{\mu-r_{l}} \omega^{\nu+r_{l}} \sum_{i=1}^{m} \mid F_{l}\left(\Lambda^{-1}(\omega) x(k+1), \Lambda^{-1}(\omega) x(k-i+1)\right) \\
-F_{l}\left(\Lambda^{-1}(\omega) x(k), \Lambda^{-1}(\omega) x(k-i+1)\right) \mid \\
=\sum_{l=1}^{n}|x(k)|_{r}^{\mu-r_{l}} \omega^{\nu+r_{l}} \sum_{i=1}^{m} \mid F_{l}\left(\Lambda^{-1}(\omega) x(k)+\Lambda^{-1}(\omega) h F(x(k), x(k-m)), \Lambda^{-1}(\omega) x(k-i+1)\right) \\
-F_{l}\left(\Lambda^{-1}(\omega) x(k), \Lambda^{-1}(\omega) x(k-i+1)\right) \mid \\
=\sum_{l=1}^{n}|x(k)|_{r}^{\mu-r_{l}} \omega^{\nu+r_{l}} \sum_{i=1}^{m} \mid F_{l}\left(\Lambda^{-1}(\omega) x(k)\right. \\
\left.+h \lambda^{\nu} \Lambda^{-1}(\omega) \Lambda(\lambda) F\left(\Lambda^{-1}(\lambda) x(k), \Lambda^{-1}(\lambda) x(k-m)\right), \Lambda^{-1}(\omega) x(k-i+1)\right) \\
-F_{l}\left(\Lambda^{-1}(\omega) x(k), \Lambda^{-1}(\omega) x(k-i+1)\right) \mid
\end{gathered}
$$




$$
\begin{aligned}
& \leq \operatorname{Lm} \sum_{l=1}^{n}|x(k)|_{r}^{\mu-r_{l}} \omega^{\nu+r_{l}} \leq L m \sum_{l=1}^{n}|x(k)|_{r}^{\mu-r_{l}} \max \left\{|x(k)+h F(x(k), x(k-m))|_{r}^{\nu+r_{l}}, \lambda^{\nu+r_{l}}\right\} \\
& \leq \operatorname{Lm} \sum_{l=1}^{n}|x(k)|_{r}^{\mu-r_{l}} \lambda^{\nu+r_{l}}\left(\left|\Lambda^{-1}(\lambda) x(k)+h \lambda^{\nu} F\left(\Lambda^{-1}(\lambda) x(k), \Lambda^{-1}(\lambda) x(k-m)\right)\right|_{r}^{\nu+r_{l}}+1\right) \\
& \leq \operatorname{Lm} \sum_{p=0}^{m}|x(k-p)|_{r}^{\nu+\mu} \sum_{l=1}^{n}\left(\left|\Lambda^{-1}(\lambda) x(k)+h \lambda^{\nu} F\left(\Lambda^{-1}(\lambda) x(k), \Lambda^{-1}(\lambda) x(k-m)\right)\right|_{r}^{\nu+r_{l}}+1\right)
\end{aligned}
$$

where the first inequality is satisfied since $\Lambda^{-1}(\omega) x(k), \Lambda^{-1}(\omega) x(k-i+1) \in \mathbb{B}_{r}$ for any $L>0$ and provided that

$$
h \lambda^{\nu}\left|\Lambda^{-1}(\omega) \Lambda(\lambda) F\left(\Lambda^{-1}(\lambda) x(k), \Lambda^{-1}(\lambda) x(k-m)\right)\right| \leq \chi
$$

for some $\chi>0$ using the uniform continuity of $F$ from Assumption 1 (we select a gain $L>0$ sufficiently small as we need, then we can find $\chi>0$ corresponding to this $L$ ). The latter property is valid if

$$
h a_{5} \lambda^{\nu} \leq \chi
$$

Therefore, combining all obtained estimates we conclude that

$$
\begin{gathered}
\Delta W \leq-a_{4} h|x(k)|_{r}^{\mu+\nu}+h \beta_{1}|x(k)|_{r}^{\rho}-h \beta_{m}|x(k-m)|_{r}^{\rho}+h \sum_{j=1}^{m-1}\left(\beta_{j+1}-\beta_{j}\right)|x(k-j)|_{r}^{\rho} \\
+4 a_{5}^{2} h^{2} \sum_{p=0}^{m}|x(k-p)|_{r}^{2 \nu+\mu} \sum_{l=1}^{n} \sum_{s=1}^{n}\left|\frac{\partial^{2} V\left(\Lambda^{-1}(\lambda) x(k)+h \theta_{2 k l} \lambda^{\nu} F\left(\Lambda^{-1}(\lambda) x(k), \Lambda^{-1}(\lambda) x(k-m)\right)\right)}{\partial z_{l} \partial z_{s}}\right| \\
+2 m a_{5}^{2} h^{2} \sum_{p=0}^{m}|x(k-p)|_{r}^{2 \nu+\mu} \sum_{l=1}^{n} \sum_{s=1}^{n}\left|\frac{\partial^{2} V\left(\Lambda^{-1}(\lambda) x(k)+h \theta_{3 k l} \lambda^{\nu} F\left(\Lambda^{-1}(\lambda) x(k), \Lambda^{-1}(\lambda) x(k-m)\right)\right)}{\partial z_{l} \partial z_{s}}\right| \\
\times\left(\left|\Lambda^{-1}(\lambda) x(k)+h \lambda^{\nu} F\left(\Lambda^{-1}(\lambda) x(k), \Lambda^{-1}(\lambda) x(k-m)\right)\right|^{\nu+r_{l}}+1\right) \\
+L m a_{6} h \sum_{p=0}^{m}|x(k-p)|_{r}^{\nu+\mu} \sum_{l=1}^{n}\left(\left|\Lambda^{-1}(\lambda) x(k)+h \lambda^{\nu} F\left(\Lambda^{-1}(\lambda) x(k), \Lambda^{-1}(\lambda) x(k-m)\right)\right|_{r}^{\nu+r_{l}}+1\right) .
\end{gathered}
$$

Recall that $\Lambda^{-1}(\lambda) x(k), \Lambda^{-1}(\lambda) x(k-m) \in \mathbb{B}_{r}$, then the following upper estimate on the increment of $W$ is derived:

$$
\begin{gathered}
\Delta W \leq-a_{4} h|x(k)|_{r}^{\mu+\nu}+h \beta_{1}|x(k)|_{r}^{\rho}-h \beta_{m}|x(k-m)|_{r}^{\rho}+h \sum_{j=1}^{m-1}\left(\beta_{j+1}-\beta_{j}\right)|x(k-j)|_{r}^{\rho} \\
+4 n^{2} a_{5}^{2} h^{2} g_{1}(\lambda) \sum_{p=0}^{m}|x(k-p)|_{r}^{2 \nu+\mu} \\
+2 m n a_{5}^{2} h^{2} g_{1}(\lambda) \sum_{p=0}^{m}|x(k-p)|_{r}^{2 \nu+\mu} \sum_{l=1}^{n}\left(g_{2}^{\nu+r_{l}}(\lambda)+1\right) \\
+L m a_{6} h \sum_{p=0}^{m}|x(k-p)|_{r}^{\nu+\mu} \sum_{l=1}^{n}\left(\left(\underline{\sigma}_{r}^{-1}\left(g_{2}(\lambda)\right)\right)^{\nu+r_{l}}+1\right)
\end{gathered}
$$

where

$$
\begin{gathered}
g_{1}(\lambda)=\max _{1 \leq l, s \leq n} \sup _{\phi, \psi \in \mathbb{B}_{r}}\left|\frac{\partial^{2} V\left(\phi+h \lambda^{\nu} F(\phi, \psi)\right)}{\partial z_{l} \partial z_{s}}\right| \\
g_{2}(\lambda)=\sup _{\phi, \psi \in \mathbb{B}_{r}}\left|\phi+h \lambda^{\nu} F(\phi, \psi)\right|
\end{gathered}
$$


and finally

$$
\begin{gathered}
\Delta W \leq-a_{4} h|x(k)|_{r}^{\mu+\nu}+h \beta_{1}|x(k)|_{r}^{\rho}-h \beta_{m}|x(k-m)|_{r}^{\rho} \\
+h \sum_{j=1}^{m-1}\left(\beta_{j+1}-\beta_{j}\right)|x(k-j)|_{r}^{\rho} \\
+h^{2} g_{3}(\lambda) \sum_{p=0}^{m}|x(k-p)|_{r}^{2 \nu+\mu}+h g_{4}(\lambda) \sum_{p=0}^{m}|x(k-p)|_{r}^{\nu+\mu},
\end{gathered}
$$

where

$$
\begin{gathered}
g_{3}(\lambda)=2 n a_{5}^{2} g_{1}(\lambda)\left[2 n+m \sum_{l=1}^{n}\left(g_{2}^{\nu+r_{l}}(\lambda)+1\right)\right], \\
g_{4}(\lambda)=L m a_{6} \sum_{l=1}^{n}\left(\left(\underline{\sigma}_{r}^{-1}\left(g_{2}(\lambda)\right)\right)^{\nu+r_{l}}+1\right) .
\end{gathered}
$$

Call back that $L$ can be chosen arbitrary small by decreasing the value of $\lambda^{\nu}$ due to (5).

\subsection{Stability results}

Now we are in position to formulate the main results of this section.

\subsubsection{Positive degree}

Theorem 1. Let assumptions 1, 2 be valid with $\nu>0, \beta_{j+1}<\beta_{j}$ for all $j=1, \ldots, m-1, \beta_{1} \leq \frac{a_{4}}{4}$ and $\rho=\mu+\nu$. Then for any $h>0$ and integer $m \geq 0$ there exists $D>0$ such that for the Lyapunov-Krasovskii functional (3) and the system (1) the relations

$$
\begin{gathered}
\frac{a_{1}}{2}|x(k)|_{r}^{\mu}+\frac{h}{2} \sum_{j=1}^{m} \beta_{j}|x(k-j)|_{r}^{\nu+\mu} \leq W \leq\left(a_{2}+2 a_{3} h n m\right)|x(k)|_{r}^{\mu} \\
+h \sum_{j=1}^{m}\left(\beta_{j}+2 a_{3} n m\right)|x(k-j)|_{r}^{\nu+\mu} \\
\Delta W \leq-\epsilon \frac{h}{2} \sum_{p=0}^{m}|x(k-p)|_{r}^{\nu+\mu}, \epsilon>0
\end{gathered}
$$

are satisfied for all $\left\|x^{(k)}\right\| \leq D$.

Proof. Note that $\mu\left(\frac{\nu}{r_{\max }}+1\right)>\nu+\mu$ since we selected $\mu>r_{\max }$, and $\rho<\mu\left(\frac{\nu}{r_{\max }}+1\right)$ due to the selection of $\rho$. Thus, using estimates (4) we obtain that

$$
\begin{gathered}
\frac{a_{1}}{2}|x(k)|_{r}^{\mu}+\frac{h}{2} \sum_{j=1}^{m} \beta_{j}|x(k-j)|_{r}^{\rho} \leq W \leq a_{2}|x(k)|_{r}^{\mu}+h \sum_{j=1}^{m} \beta_{j}|x(k-j)|_{r}^{\rho} \\
+2 a_{3} h n m \sum_{p=0}^{m}|x(k-p)|_{r}^{\nu+\mu}
\end{gathered}
$$

for $\left\|x^{(k)}\right\| \leq D_{1}$ with

$$
\begin{aligned}
D_{1} \leq \min _{1 \leq j \leq n}\left\{\left(\frac{a_{1}}{4 a_{3} h n m}\right)^{\nu^{-1}},\left(\frac{2 n a_{3} h^{\frac{\rho-\nu-r_{j}}{\rho}}}{\min \left\{\frac{a_{1}}{2 m}, \beta_{j}\right\}}\right)^{\frac{\mu^{-1}}{1-\frac{\mu-r_{j}}{\mu}-\frac{\nu+r_{j}}{\rho}}},\right. \\
\left.h^{-\rho^{-1}}\left(\frac{2 n a_{3} h^{\frac{\rho-\nu-r_{j}}{\rho}}}{\min \left\{\frac{a_{1}}{2 m}, \beta_{j}\right\}}\right)^{\frac{\rho^{-1}}{1-\frac{\mu-r_{j}}{\mu}-\frac{\nu+r_{j}}{\rho}}}\right\}
\end{aligned}
$$


where the computations for the upper estimate are straightforward (it holds without a restriction on $\left\|x^{(k)}\right\|$ ) and Lemma 4 was used for the lower one. Note that for $\nu>0$, for any $D_{2}>0$ the functions $g_{1}(\lambda)$ and $g_{2}(\lambda)$ admit an upper bound for all $\lambda=\left\|x^{(k)}\right\| \leq D_{2}$ and

$$
g_{1}(\lambda) \geq a_{7}=\max _{1 \leq l, s \leq n} \sup _{\phi \in \mathbb{B}_{r}}\left|\frac{\partial^{2} V(\phi)}{\partial z_{l} \partial z_{s}}\right|, g_{2}(\lambda) \geq a_{8}=\sup _{\phi \in \mathbb{B}_{r}}|\phi|,
$$

then

$$
\begin{gathered}
a_{9} \leq g_{3}(\lambda) \leq \bar{g}_{3}\left(D_{2}\right), a_{10} \leq g_{4}(\lambda) \leq \bar{g}_{4}\left(D_{2}\right), \\
a_{9}=2 n a_{5}^{2} a_{7}\left[2 n+m \sum_{l=1}^{n}\left(a_{8}^{\nu+r_{l}}+1\right)\right], \\
a_{10}=L m a_{5}^{\chi} a_{6} \sum_{l=1}^{n}\left(\left(\underline{\sigma}_{r}^{-1}\left(a_{8}\right)\right)^{\nu+r_{l}}+1\right)
\end{gathered}
$$

for all $\left\|x^{(k)}\right\| \leq D_{2}$ (and additionally $L>0$ can be made arbitrary small by decreasing the value of $D_{2}$ using $(5)$ ), where $\bar{g}_{3}\left(D_{2}\right)$ and $\bar{g}_{4}\left(D_{2}\right)$ are the respective upper bounds for $g_{3}$ and $g_{4}$. For $\rho=\nu+\mu$ and any $D_{2}>0$, the estimate (6) can be rewritten as follows:

$$
\Delta W \leq-\frac{h}{2}\left(a_{4}|x(k)|_{r}^{\mu+\nu}+\beta_{m}|x(k-m)|_{r}^{\rho}+\sum_{j=1}^{m-1}\left(\beta_{j}-\beta_{j+1}\right)|x(k-j)|_{r}^{\rho}\right)
$$

for all $\left\|x^{(k)}\right\| \leq D_{2}$, which is a solution of the inequality

$$
D_{2} \leq\left(\frac{\min \left\{\frac{a_{4}}{2}, \beta_{m}, \beta_{1}-\beta_{2}, \ldots, \beta_{m-1}-\beta_{m}\right\}}{4 h \bar{g}_{3}\left(D_{2}\right)}\right)^{\nu^{-1}},
$$

and for

$$
\beta_{1} \leq \frac{a_{4}}{4}, \bar{g}_{4}\left(D_{2}\right) \leq \frac{\min \left\{\frac{a_{4}}{2}, \beta_{m}, \beta_{1}-\beta_{2}, \ldots, \beta_{m-1}-\beta_{m}\right\}}{4} .
$$

These inequalities always have a solution with respect to $D_{2}$ since for $D_{2} \rightarrow 0$ the right-hand side of the former stays separated from zero converging to

$$
\left(\frac{\min \left\{\frac{a_{4}}{2}, \beta_{m}, \beta_{1}-\beta_{2}, \ldots, \beta_{m-1}-\beta_{m}\right\}}{4 h a_{9}}\right)^{\nu^{-1}},
$$

while $\bar{g}_{4}\left(D_{2}\right)$ can be made as small as needed with $D_{2} \rightarrow 0$ recalling (5). Thus, with $D=\min \left\{D_{1}, D_{2}\right\}$ and $\epsilon=\min \left\{a_{4}, \beta_{m}, \beta_{1}-\beta_{2}, \ldots, \beta_{m-1}-\beta_{m}\right\}$ the statements of the theorem are verified.

An approach to evaluate the values of $D$ (the bound on domain of positive definiteness of $W$ and negative definiteness of $\Delta W$ ) and $\epsilon$ (the convergence rate) is suggested in the proof of Theorem 1.

Remark 1. As we can conclude from the proof of Theorem 1 , if $h \rightarrow+\infty$ or $m \rightarrow+\infty$, then $D \rightarrow 0$ (since $\left.1-\frac{\mu-r_{j}}{\mu}-\frac{\nu+r_{j}}{\rho}<0\right)$, which corresponds to the results obtained in $[20,39]$ that with the growth of the delay, the domain of convergence is shrinking.

Remark 2. If we assume that $F$ is $\chi^{\prime}$-Hölder continuous on $\mathbb{B}_{r}$ with constant $L^{\prime}>0$ with respect to the first argument, $\chi^{\prime} \in(0,1]$, and for $\mu>\left(1+\chi^{\prime}\right) r_{\max }$ (see also Lemma 3), then the conclusion of Theorem 1 can be obtained for $\mu+\nu<\rho<\left(1+\chi^{\prime}\right) \nu+\mu$ and without a restriction on $\beta_{1}$.

Using the result of Lemma 1 the following time estimate on decay of solutions in (1) can be obtained:

Corollary 1. Let all conditions of Theorem 1 be valid, then there exist $\delta>0$ and $\alpha>0$ such that

$$
W\left(x^{(k)}\left(x^{(0)}\right)\right) \leq W\left(x^{(0)}\right)\left(1+\alpha \frac{\nu}{\mu} W^{\frac{\nu}{\mu}}\left(x^{(0)}\right) k\right)^{-\frac{\mu}{\nu}}
$$

for all $k \geq 0$ and all $\left\|x^{(0)}\right\| \leq \delta$. 
Proof. Consider the Lyapunov-Krasovskii functional (3) constructed for the system (1) in Theorem 1. Since all conditions of the theorem are valid, there exists $D>0$ such that for all $\left\|x^{(k)}\right\| \leq D$ :

$$
\begin{gathered}
W^{1+\frac{\nu}{\mu}} \leq c\left(|x(k)|_{r}^{\mu+\nu}+\sum_{j=1}^{m}|x(k-j)|_{r}^{\frac{(\nu+\mu)^{2}}{\mu}}\right) \\
\leq c^{\prime} \sum_{p=0}^{m}|x(k-p)|_{r}^{\nu+\mu},
\end{gathered}
$$

where

$$
\begin{gathered}
c=(2 m+2)^{\frac{\nu}{\mu}}\left(\max \left\{a_{2}, h \max _{1 \leq j \leq m} \beta_{j}\right\}+2 a_{3} h n m\right)^{1+\frac{\nu}{\mu}}, \\
c^{\prime}=c \max \left\{1, D^{\nu+\frac{\nu^{2}}{\mu}}\right\},
\end{gathered}
$$

and Jensen's inequality was used on the first step, while simple relation $s^{\eta+\varpi} \leq D^{\varpi} s^{\eta}$, which is true for any $s \in[0, D]$ and $\eta, \varpi>0$, was utilized on the last one. Therefore,

$$
W\left(x^{(k+1)}\right) \leq W\left(x^{(k)}\right)-\alpha W^{1+\frac{\nu}{\mu}}\left(x^{(k)}\right),
$$

where $\alpha=\epsilon \frac{h}{2 c^{\prime}}$. Choose $\delta>0$ such that if $\left\|x^{(0)}\right\| \leq \delta$, then $\left\|x^{(k)}\left(x^{(0)}\right)\right\| \leq D$ for $k \geq 0$ and $\alpha\left(1+\frac{\nu}{\mu}\right) W^{\frac{\nu}{\mu}}\left(x^{(0)}\right) \leq 1$, and with the aid of Lemma 1 we obtain the required estimate.

Note that $\frac{a_{1}}{2}|x(k)|_{r}^{\mu} \leq W\left(x^{(k)}\right)$, then the time rate of convergence of $x(k)$ is of order $k^{-\nu^{-1}}$, which is the same as for the continuous-time delay-free system.

\subsubsection{Negative degree}

For this part we introduce an additional restriction on $V$ and assume that $\mu>\max \left\{r_{\max }, 1+r_{\min }\right\}$.

Theorem 2. Let assumptions 1, 2 be valid with $-r_{\min }<\nu<0, \beta_{j+1}<\beta_{j}$ for all $j=1, \ldots, m-1, \beta_{1}<\frac{a_{4}}{4}$ and $\rho=\mu+\nu$. Then for any $h>0$ and integer $m \geq 0$ there exists $D>0$ such that for the Lyapunov-Krasovskii functional (3) and the system (1) the relations

$$
\begin{gathered}
\frac{a_{1}}{2}|x(k)|_{r}^{\mu}+\frac{h}{2} \sum_{j=1}^{m} \beta_{j}|x(k-j)|_{r}^{\nu+\mu} \leq W \leq\left(a_{2}+2 a_{3} h n m\right)|x(k)|_{r}^{\mu} \\
+h \sum_{j=1}^{m}\left(\beta_{j}+2 a_{3} n m\right)|x(k-j)|_{r}^{\nu+\mu} \\
\Delta W \leq-\epsilon \frac{h}{2} \sum_{p=0}^{m}|x(k-p)|_{r}^{\nu+\mu}, \epsilon>0
\end{gathered}
$$

are satisfied for all $\left\|x^{(k)}\right\| \geq D$.

Proof. Note that $\mu\left(\frac{\nu}{r_{\max }}+1\right)<\nu+\mu$ since we selected $\mu>r_{\max }$ and $\nu<0$, and $\rho>\mu\left(\frac{\nu}{r_{\max }}+1\right)$ due to the selection of $\rho$. Thus, using (4) the upper estimate can be derived in the same way as in Theorem 1 , and the lower estimate holds provided that

$$
\begin{gathered}
\min \left\{a_{1}, \beta_{\min }\right\} \max \left\{|x(k)|_{r}^{\mu}, h \sum_{i=1}^{m}|x(k-i)|_{r}^{\rho}\right\} \\
\geq 2 a_{3} h\left(n m|x(k)|_{r}^{\nu+\mu}+\sum_{j=1}^{n}|x(k)|_{r}^{\mu-r_{j}} \sum_{i=1}^{m}|x(k-i)|_{r}^{\nu+r_{j}}\right),
\end{gathered}
$$

where $\beta_{\min }=\min \left\{\beta_{1}, \ldots, \beta_{m}\right\}$. Defining $d_{1}=\frac{4 a_{3} h}{\min \left\{a_{1}, \beta_{\min }\right\}}$ this inequality can be decomposed on

$$
\begin{gathered}
\max \left\{|x(k)|_{r}^{\mu}, h \sum_{i=1}^{m}|x(k-i)|_{r}^{\rho}\right\} \geq d_{1} n m|x(k)|_{r}^{\nu+\mu} \\
\max \left\{|x(k)|_{r}^{\mu}, h \sum_{i=1}^{m}|x(k-i)|_{r}^{\rho}\right\} \geq d_{1} \sum_{j=1}^{n}|x(k)|_{r}^{\mu-r_{j}} \sum_{i=1}^{m}|x(k-i)|_{r}^{\nu+r_{j}} .
\end{gathered}
$$


This former can be resolved directly, and Lemma 4 can be used for the latter since

$$
\sum_{i=1}^{m}|x(k-i)|_{r}^{\nu+r_{j}} \leq m^{1-\frac{\nu+r_{j}}{\rho}}\left(\sum_{i=1}^{m}|x(k-i)|_{r}^{\rho}\right)^{\frac{\nu+r_{j}}{\rho}}
$$

by applying Jensen's inequality for $\mu>r_{\max }$. Hence, these inequalities are valid for

$$
\begin{gathered}
\max \left\{|x(k)|_{r}^{\mu}, h \sum_{i=1}^{m}|x(k-i)|_{r}^{\rho}\right\} \geq \max _{1 \leq j \leq n}\left\{\left(d_{1} n m\right)^{-\nu^{-1}},\right. \\
\left.\left(2 d_{1} n m(h m)^{-\frac{\nu+r_{j}}{\rho}}\right)^{\frac{1}{1-\frac{\mu-r_{j}}{\mu}-\frac{\nu+r_{j}}{\rho}}},\left(d_{1} n m\right)^{-\frac{\mu}{\nu}}\right\},
\end{gathered}
$$

and there exists $D_{1}>0$ such that $\left\|x^{(k)}\right\| \geq D_{1}$ is included in the above domain. Note that for $\nu<0$, for any $D_{2}>0$ the functions $g_{1}(\lambda)$ and $g_{2}(\lambda)$ admit an upper bound for all $\lambda=\left\|x^{(k)}\right\| \geq D_{2}$ and

$$
g_{1}(\lambda) \geq a_{7}, g_{2}(\lambda) \geq a_{8},
$$

then

$$
a_{9} \leq g_{3}(\lambda) \leq \bar{g}_{3}\left(D_{2}\right), a_{10} \leq g_{4}(\lambda) \leq \bar{g}_{4}\left(D_{2}\right),
$$

for all $\left\|x^{(k)}\right\| \geq D_{2}$, where $\bar{g}_{3}\left(D_{2}\right)$ and $\bar{g}_{4}\left(D_{2}\right)$ are the respective upper bounds for $g_{3}$ and $g_{4}$, and the constants $a_{7}, a_{8}, a_{9}$ and $a_{10}$ are defined in the proof of Theorem 1 (similarly, $\bar{g}_{4}\left(D_{2}\right)$ is proportional to $L$ that can be made arbitrary small by increasing the value of $D_{2}$ using (5)). For any $D_{2}>0$, the estimate (6) can be rewritten as follows:

$$
\Delta W \leq-\frac{h}{2}\left(a_{4}|x(k)|_{r}^{\mu+\nu}+\beta_{m}|x(k-m)|_{r}^{\rho}+\sum_{j=1}^{m-1}\left(\beta_{j}-\beta_{j+1}\right)|x(k-j)|_{r}^{\rho}\right)
$$

provided that

$$
\begin{gathered}
\frac{1}{2}\left(a_{4}|x(k)|_{r}^{\mu+\nu}+\beta_{m}|x(k-m)|_{r}^{\rho}+\sum_{j=1}^{m-1}\left(\beta_{j}-\beta_{j+1}\right)|x(k-j)|_{r}^{\rho}\right) \geq \beta_{1}|x(k)|_{r}^{\rho} \\
+h \bar{g}_{3}\left(D_{2}\right) \sum_{p=0}^{m}|x(k-p)|_{r}^{2 \nu+\mu}+\bar{g}_{4}\left(D_{2}\right) \sum_{p=0}^{m}|x(k-p)|_{r}^{\nu+\mu} .
\end{gathered}
$$

Since $\rho=\nu+\mu$, verifying the terms with the same powers leads to:

$$
\beta_{1}+\bar{g}_{4}\left(D_{2}\right) \leq \frac{a_{4}}{4}, \bar{g}_{4}\left(D_{2}\right) \leq \frac{\min \left\{\beta_{m}, \beta_{1}-\beta_{2}, \ldots, \beta_{m-1}-\beta_{m}\right\}}{4},
$$

which can be ensured by increasing the value of $D_{2}$ as it was explained above. Then the last property to establish is

$$
\sum_{p=0}^{m}|x(k-j)|_{r}^{\nu+\mu} \geq d_{2} \sum_{p=0}^{m}|x(k-p)|_{r}^{2 \nu+\mu}
$$

for

$$
d_{2}=\frac{4 h \bar{g}_{3}\left(D_{2}\right)}{\min \left\{a_{4}, \beta_{m}, \beta_{1}-\beta_{2}, \ldots, \beta_{m-1}-\beta_{m}\right\}} .
$$

Applying Jensen's inequality we get

$$
\sum_{p=0}^{m}|x(k-p)|_{r}^{2 \nu+\mu} \leq(m+1)^{-\frac{\nu}{\nu+\mu}}\left(\sum_{p=0}^{m}|x(k-j)|_{r}^{\nu+\mu}\right)^{\frac{2 \nu+\mu}{\nu+\mu}}
$$

due to $\nu+\mu \geq 1$ for $\mu>1+r_{\min }$, then

$$
\sum_{p=0}^{m}|x(k-j)|_{r}^{\nu+\mu} \geq(m+1) d_{2}^{-\frac{\nu+\mu}{\nu}}
$$

is the condition to check, and using again Jensen's inequality we can show that there exists $D_{2}>0$ such that the above relation is satisfied for $\left\|x^{(k)}\right\| \geq D_{2}$ (as in the proof of Theorem 1, increasing the value of $D_{2}$ will push $\bar{g}_{3}\left(D_{2}\right)$ to its lower bound $\left.a_{9}\right)$. Thus, with $D=\max \left\{D_{1}, D_{2}\right\}$ and $\epsilon=\min \left\{a_{4}, \beta_{m}, \beta_{1}-\beta_{2}, \ldots, \beta_{m-1}-\beta_{m}\right\}$ the statements of the theorem are verified. 
Remark 3. For $h \rightarrow+\infty$ or $m \rightarrow+\infty$ the constants $d_{1}$ and $d_{2}$, whose values are given in the proof of Theorem 2 , will also grow to infinity implying the same property of $D$ (it is a function of $d_{1}$ and $d_{2}$ ). This result for the continuous-time systems was established in [30,39]. Similarly to the continuous-time case, in theorems 1 or 2 , $h \rightarrow 0$ implies the growth of $D$ to infinity $(\nu>0)$ or its decay to zero $(\nu<0)$.

Corollary 2. Let all conditions of Theorem 2 be valid, then there exist positive numbers $\delta_{1}, \delta_{2}$ and $\alpha$ such that

$$
W\left(x^{(k)}\left(x^{(0)}\right)\right) \leq \max \left\{\delta_{2}, W\left(x^{(0)}\right)\left(1+\alpha \frac{\nu}{\mu} W^{\frac{\nu}{\mu}}\left(x^{(0)}\right) k\right)^{-\frac{\mu}{\nu}}\right\}
$$

for all $k \geq 0$ and all $\left\|x^{(0)}\right\| \geq \delta_{1}$.

Proof. Consider the Lyapunov-Krasovskii functional (3) constructed for the system (1) in Theorem 2. Since all conditions of the theorem are valid, there exists $D>0$ such that for all $\left\|x^{(k)}\right\| \geq D$ :

$$
\begin{aligned}
& W \leq c\left(|x(k)|_{r}^{\mu}+\sum_{j=1}^{m}|x(k-j)|_{r}^{\nu+\mu}\right) \leq c(m+1) \max \left\{\left\|x^{(k)}\right\|^{\mu},\left\|x^{(k)}\right\|^{\nu+\mu}\right\}, \\
& W^{1+\frac{\nu}{\mu}} \leq c^{1+\frac{\nu}{\mu}}\left(|x(k)|_{r}^{\mu+\nu}+\sum_{j=1}^{m}|x(k-j)|_{r}^{\frac{(\nu+\mu)^{2}}{\mu}}\right) \leq c^{\prime} \sum_{p=0}^{m}|x(k-p)|_{r}^{\nu+\mu},
\end{aligned}
$$

where

$$
\begin{gathered}
c=\max \left\{a_{2}, h \max _{1 \leq j \leq m} \beta_{j}\right\}+2 a_{3} h n m, \\
c^{\prime}=c^{1+\frac{\nu}{\mu}} \max \left\{1, D^{\frac{\nu(\nu+\mu)}{\mu}}\right\},
\end{gathered}
$$

and inequality $s^{\eta-\varpi} \leq D^{-\varpi} s^{\eta}$ was used in the second case, which is true for any $s \geq D$ and $\eta$, $>0$. Therefore,

$$
W\left(x^{(k+1)}\right) \leq W\left(x^{(k)}\right)-\alpha W^{1+\frac{\nu}{\mu}}\left(x^{(k)}\right),
$$

where $\alpha=\epsilon \frac{h}{2 c^{\prime}}$. Choose $\delta_{1}>0$ such that $W\left(x^{(0)}\right)>\max \left\{c(m+1) \max \left\{D^{\mu}, D^{\nu+\mu}\right\}, \alpha^{-\frac{\mu}{\nu}}\right\}$ for $\left\|x^{(0)}\right\| \geq \delta_{1}$, then with the aid of Lemma 2 we obtain the estimate

$$
W\left(x^{(k)}\left(x^{(0)}\right)\right) \leq W\left(x^{(0)}\right)\left(1+\alpha \frac{\nu}{\mu} W^{\frac{\nu}{\mu}}\left(x^{(0)}\right) k\right)^{-\frac{\mu}{\nu}}
$$

for all $k=0,1, \ldots, N$, where $N \leq N_{0}=-\frac{\nu}{\mu} \frac{W^{-\frac{\nu}{\mu}}\left(x^{(0)}\right)-\alpha}{\alpha}$. From another side, $W\left(x^{(k+1)}\right) \leq W\left(x^{(k)}\right)$ while $W\left(x^{(k)}\right)>c(m+1) \max \left\{D^{\mu}, D^{\nu+\mu}\right\}=\delta_{2}$, which leads to the proposed estimate.

Remark 4. The obtained results in this section can be extended to a homogeneous system with multiple delays:

$$
x(k+1)=x(k)+h F(x(k), x(k-1), x(k-2), \ldots, x(k-m)) .
$$

Due to properties of the Lyapunov-Krasovskii functional (3), the results of corollaries 1 and 2 imply asymptotic stability of the zero solution of (1) with the domain of attraction $\left\|x^{(0)}\right\| \leq \delta$ and convergence of all trajectories of (1) with initial conditions in $\left\{\xi \in \mathbb{R}^{n(m+1)}:\|\xi\| \leq \delta_{1}\right\}$ to the set $\left\{\xi \in \mathbb{R}^{n(m+1)}: W(\xi) \leq \delta_{2}\right\}$ $[2,3]$, respectively.

\section{$4 \quad$ Perturbed System}

Consider a perturbed version of the system (1):

$$
x(k+1)=x(k)+h F(x(k), x(k-m))+h Q\left(k, x^{(k)}\right),
$$

where $Q: \mathbb{R}_{+} \times \mathbb{R}^{n(m+1)} \rightarrow \mathbb{R}^{n}$ is a locally bounded function, which satisfies the condition:

Assumption 3. For all $x^{(k)} \in \mathbb{R}^{n(m+1)}, k \geq 0$ and $i=1, \ldots, n$,

$$
\left|Q_{i}\left(k, x^{(k)}\right)\right| \leq c \sum_{p=0}^{m}|x(k-p)|_{r}^{\sigma+r_{i}}
$$

for some $c>0$ and $\sigma \geq-r_{\min }$. 
Since as in theorems 1 or 2 , the analysis will be performed either for $\left\|x^{(k)}\right\| \leq D(\nu>0)$ or for $\left\|x^{(k)}\right\| \geq D$ $(\nu<0)$, then the above hypothesis we will not need globally, and this formulation is given for all $\mathbb{R}^{n(m+1)}$ for brevity only.

Remark 5. Under Assumption 3, the system (7) admits homogeneous approximations at the origin or at infinity in the form of (1) for $\sigma>\nu>0$ or $\sigma<\nu<0$, respectively. These scenarios will be analyzed in the sequel of the section.

To study the stability of (7), the Lyapunov-Krasovskii functional (3) can again be applied. The lower and upper estimates (4) save their meaning. Hence, consider the increment of the functional $W$ along the solutions of (7):

$$
\begin{gathered}
\Delta W=h\left(\frac{\partial V\left(x(k)+\theta_{1 k} \Delta x(k)\right)}{\partial z}\right)^{T}\left(F(x(k), x(k-m))+Q\left(k, x^{(k)}\right)\right) \\
+h \sum_{j=1}^{m} \beta_{j}|x(k-j+1)|_{r}^{\rho}-h \sum_{j=1}^{m} \beta_{j}|x(k-j)|_{r}^{\rho} \\
+h\left(\frac{\partial V(x(k)+\Delta x(k))}{\partial z}\right)^{T} \sum_{i=1}^{m} F(x(k+1), x(k-i+1))-h\left(\frac{\partial V(x(k))}{\partial z}\right)^{T} \sum_{i=1}^{m} F(x(k), x(k-i)) \\
=h\left(\frac{\partial V(x(k))}{\partial z}\right)^{T} F(x(k), x(k))+h\left(\frac{\partial V(x(k))}{\partial z}\right)^{T} Q\left(k, x^{(k)}\right) \\
+h\left(\frac{\partial V\left(x(k)+\theta_{1 k} \Delta x(k)\right)}{\partial z}-\frac{\partial V(x(k))}{\partial z}\right)^{T}\left(F(x(k), x(k-m))+Q\left(k, x^{(k)}\right)\right) \\
+h \beta_{1}|x(k)|_{r}^{\rho}-h \beta_{m}|x(k-m)|_{r}^{\rho}+h \sum_{j=1}^{m-1}\left(\beta_{j+1}-\beta_{j}\right)|x(k-j)|_{r}^{\rho} \\
+h\left(\frac{\partial V(x(k))}{\partial z}\right)^{T}\left(\sum_{i=1}^{m} F(x(k+1), x(k-i+1))-\sum_{i=1}^{m} F(x(k), x(k-i+1))\right)
\end{gathered}
$$

where $\Delta x(k)=h\left(F(x(k), x(k-m))+Q\left(k, x^{(k)}\right)\right), \theta_{1 k} \in(0,1)$ and we used the same tricks as before. Applying Assumption 3 and since (as previously, denote $\lambda=\left\|x^{(k)}\right\|$ for brevity)

$$
\begin{gathered}
\left|\left(\frac{\partial V(x(k))}{\partial z}\right)^{T} Q\left(k, x^{(k)}\right)\right| \leq a_{6} c \sum_{i=1}^{n}|x(k)|_{r}^{\mu-r_{i}} \sum_{p=0}^{m}|x(k-p)|_{r}^{\sigma+r_{i}} \\
\leq a_{6} c \sum_{i=1}^{n} \lambda^{\mu-r_{i}} \sum_{p=0}^{m} \lambda^{\sigma+r_{i}} \leq a_{6} c(m+1) n \sum_{p=0}^{m}|x(k-p)|_{r}^{\sigma+\mu}
\end{gathered}
$$

we obtain:

$$
\begin{gathered}
\Delta W \leq-a_{4} h|x(k)|_{r}^{\mu+\nu}+h \beta_{1}|x(k)|_{r}^{\rho}-h \beta_{m}|x(k-m)|_{r}^{\rho}+h \sum_{j=1}^{m-1}\left(\beta_{j+1}-\beta_{j}\right)|x(k-j)|_{r}^{\rho} \\
+h a_{6} c(m+1) n \sum_{p=0}^{m}|x(k-p)|_{r}^{\sigma+\mu} \\
+h \max \left\{a_{5}, c\right\} \sum_{l=1}^{n}\left|\frac{\partial V\left(x(k)+\theta_{1 k} \Delta x(k)\right)}{\partial z_{l}}-\frac{\partial V(x(k))}{\partial z_{l}}\right|\left(|x(k)|_{r}^{\nu+r_{l}}+|x(k-m)|_{r}^{\nu+r_{l}}+\sum_{p=0}^{m}|x(k-p)|_{r}^{\sigma+r_{l}}\right) \\
+h a_{5} \sum_{l=1}^{n}\left|\frac{\partial V(x(k)+\Delta x(k))}{\partial z_{l}}-\frac{\partial V(x(k))}{\partial z_{l}}\right| \sum_{i=1}^{m}\left(|x(k+1)|_{r}^{\nu+r_{l}}+|x(k-i+1)|_{r}^{\nu+r_{l}}\right) \\
+h a_{6} \sum_{l=1}^{n}|x(k)|_{r}^{\mu-r_{l}} \sum_{i=1}^{m}\left|F_{l}(x(k+1), x(k-i+1))-F_{l}(x(k), x(k-i+1))\right|
\end{gathered}
$$


Repeating the same operations as in the previous section and using the equality

$$
Q\left(k, x^{(k)}\right)=\lambda^{\sigma} \Lambda(\lambda) \varsigma_{k}
$$

with $\varsigma_{k} \in \Gamma=\left\{z \in \mathbb{R}^{n}:|z| \leq(m+1) c\right\}$ we derive:

$$
\begin{aligned}
& \left|\frac{\partial V\left(x(k)+\theta_{1 k} \Delta x(k)\right)}{\partial z_{l}}-\frac{\partial V(x(k))}{\partial z_{l}}\right|\left(|x(k)|_{r}^{\nu+r_{l}}+|x(k-m)|_{r}^{\nu+r_{l}}+\sum_{p=0}^{m}|x(k-p)|_{r}^{\sigma+r_{l}}\right) \\
& \leq h w_{1}\left(\sum_{p=0}^{m}|x(k-p)|_{r}^{2 \nu+\mu}+\sum_{p=0}^{m}|x(k-p)|_{r}^{\sigma+\nu+\mu}+\sum_{p=0}^{m}|x(k-p)|_{r}^{2 \sigma+\mu}\right) \\
& \times \sum_{s=1}^{n}\left|\frac{\partial^{2} V\left(\Lambda^{-1}(\lambda) x(k)+h \theta_{2 k l}\left[\lambda^{\nu} F\left(\Lambda^{-1}(\lambda) x(k), \Lambda^{-1}(\lambda) x(k-m)\right)+\lambda^{\sigma} \varsigma_{k}\right]\right)}{\partial z_{l} \partial z_{s}}\right|, \\
& \left|\frac{\partial V(x(k)+\Delta x(k))}{\partial z_{l}}-\frac{\partial V(x(k))}{\partial z_{l}}\right| \sum_{i=1}^{m}\left(|x(k+1)|_{r}^{\nu+r_{l}}+|x(k-i+1)|_{r}^{\nu+r_{l}}\right) \\
& \leq m \max \left\{a_{5}, c\right\} h\left(2 \sum_{p=0}^{m}|x(k-p)|_{r}^{2 \nu+\mu}+(m+1) \sum_{p=0}^{m}|x(k-p)|_{r}^{\sigma+\nu+\mu}\right) \\
& \times \sum_{s=1}^{n}\left|\frac{\partial^{2} V\left(\Lambda^{-1}(\lambda) x(k)+h \theta_{3 k l}\left[\lambda^{\nu} F\left(\Lambda^{-1}(\lambda) x(k), \Lambda^{-1}(\lambda) x(k-m)\right)+\lambda^{\sigma} \varsigma_{k}\right]\right)}{\partial z_{l} \partial z_{s}}\right| \\
& \times\left(\left|\Lambda^{-1}(\lambda) x(k)+h\left[\lambda^{\nu} F\left(\Lambda^{-1}(\lambda) x(k), \Lambda^{-1}(\lambda) x(k-m)\right)+\lambda^{\sigma} \varsigma_{k}\right]\right|^{\nu+r_{l}}+1\right), \\
& \sum_{l=1}^{n}|x(k)|_{r}^{\mu-r_{l}} \sum_{i=1}^{m}\left|F_{l}(x(k+1), x(k-i+1))-F_{l}(x(k), x(k-i+1))\right| \\
& \leq \operatorname{Lm} \sum_{p=0}^{m}|x(k-p)|_{r}^{\nu+\mu} \sum_{l=1}^{n}\left(\left|\Lambda^{-1}(\lambda) x(k)+h\left[\lambda^{\nu} F\left(\Lambda^{-1}(\lambda) x(k), \Lambda^{-1}(\lambda) x(k-m)\right)+\lambda^{\sigma} \varsigma\right]\right|_{r}^{\nu+r_{l}}+1\right),
\end{aligned}
$$

where $\theta_{2 k l}, \theta_{3 k l} \in(0,1)$,

$$
w_{1}=\max \left\{a_{5}, c\right\} \max \left\{4,4(m+1),(m+1)^{2}\right\},
$$

and for any $L>0$ the latter inequality is satisfied under the constraint

$$
h\left[a_{5} \lambda^{\nu}+\lambda^{\sigma}(m+1) c\right] \leq \chi
$$

with some $\chi>0$ due to uniform continuity of $F$ from Assumption 1. Combining with the estimates calculated in the previous section we conclude:

$$
\begin{gathered}
\Delta W \leq-a_{4} h|x(k)|_{r}^{\mu+\nu}+h \beta_{1}|x(k)|_{r}^{\rho}-h \beta_{m}|x(k-m)|_{r}^{\rho}+h \sum_{j=1}^{m-1}\left(\beta_{j+1}-\beta_{j}\right)|x(k-j)|_{r}^{\rho} \\
+h a_{6} c(m+1) n \sum_{p=0}^{m}|x(k-p)|_{r}^{\sigma+\mu}+h g_{9}(\lambda) \sum_{p=0}^{m}|x(k-p)|_{r}^{\nu+\mu}+h^{2}\left(g_{7}(\lambda)+2 g_{8}(\lambda)\right) \sum_{p=0}^{m}|x(k-p)|_{r}^{2 \nu+\mu} \\
+h^{2}\left(g_{7}(\lambda)+(m+1) g_{8}(\lambda)\right) \sum_{p=0}^{m}|x(k-p)|_{r}^{\sigma+\nu+\mu}+h^{2} g_{7}(\lambda) \sum_{p=0}^{m}|x(k-p)|_{r}^{2 \sigma+\mu}
\end{gathered}
$$

where

$$
\begin{gathered}
g_{5, l}(\lambda)=\sup _{\phi, \psi \in \mathbb{B}_{r}} \sup _{\varsigma \in \Gamma} \sum_{s=1}^{n}\left|\frac{\partial^{2} V\left(\phi+h\left[\lambda^{\nu} F(\phi, \psi)+\lambda^{\sigma} \varsigma\right]\right)}{\partial z_{l} \partial z_{s}}\right|, \\
g_{6}(\lambda)=\sup _{\phi, \psi \in \mathbb{B}_{r}} \sup _{\varsigma \in \Gamma}\left|\phi+h\left[\lambda^{\nu} F(\phi, \psi)+\lambda^{\sigma} \varsigma_{k}\right]\right| \\
g_{7}(\lambda)=\max \left\{a_{5}, c\right\} w_{1} \sum_{l=1}^{n} g_{5, l}(\lambda), g_{8}(\lambda)=a_{5} m \max \left\{a_{5}, c\right\} \sum_{l=1}^{n}\left(g_{6}^{\nu+r_{l}}(\lambda)+1\right) g_{5, l}(\lambda), \\
g_{9}(\lambda)=L a_{6} m \sum_{l=1}^{n}\left(\left(\underline{\sigma}_{r}^{-1}\left(g_{6}(\lambda)\right)\right)^{\nu+r_{l}}+1\right) .
\end{gathered}
$$


Thus, in (9) with respect to (6), there are three additional terms containing the powers proportional to $\sigma$ (their appearance is related with the disturbing term $Q)$.

Theorem 3. Let assumptions 1, 2 and 3 be valid for $\sigma>\nu>0$. Then for any $h>0$ and integer $m \geq 0$ there exists $D>0$ such that the zero solution of (7) is asymptotically stable for all $\left\|x^{(0)}\right\| \leq D$.

Proof. The proof of this result, first, follows the steps of the proof of Theorem 1 to establish the same properties of $W$ with $\beta_{j+1}<\beta_{j}$ for all $j=1, \ldots, m-1, \beta_{1} \leq \frac{a_{4}}{4}$ and $\rho=\mu+\nu$ (the terms containing the powers with $\sigma$, since $\sigma>\nu$, can be treated as ones with $\mu+2 \nu$ previously). Second, the result of Corollary 1 can be used to prove asymptotic convergence to the origin.

Theorem 4. Let assumptions 1, 2 and 3 be valid for $-r_{\min } \leq \sigma<\nu<0$. Then for any $h>0$ and integer $m \geq 0$ there exists $D>0$ such that all trajectories of (7) with $\left\|x^{(0)}\right\| \geq D$ converge to the set $\left\{\xi \in \mathbb{R}^{n(m+1)}:\|\xi\| \leq \bar{D}\right\}$.

Proof. The proof is based on application of the arguments used in the proof of Theorem 2 (for $W$ with $\beta_{j+1}<\beta_{j}$ for all $j=1, \ldots, m-1, \beta_{1}<\frac{a_{4}}{4}$ and $\left.\rho=\mu+\nu\right)$ and on Corollary 2.

\section{Simulations}

Consider a double integrator nominal system:

$$
\begin{gathered}
\dot{x}_{1}(t)=x_{2}(t)+Q_{1}(t, x(t), x(t-h), \ldots, x(t-m h)), \\
\dot{x}_{2}(t)=u(t)+Q_{2}(t, x(t), x(t-h), \ldots, x(t-m h)),
\end{gathered}
$$

where $x_{1}(t), x_{2}(t) \in \mathbb{R}, x(t)=\left[x_{1}(t), x_{2}(t)\right]^{\top}, u(t) \in \mathbb{R}$ is the control, $t \geq 0, Q_{1}, Q_{2}: \mathbb{R}^{m+2} \rightarrow \mathbb{R}$ are the disturbances. This system can be stabilized by a feedback [14]:

$$
u(t)=-k_{1}\left|x_{1}(t-m h)\right|^{1+2 \nu} \operatorname{sign}\left(x_{1}(t-m h)\right)-k_{2}\left|x_{2}(t-m h)\right|^{\frac{1+2 \nu}{1+\nu}} \operatorname{sign}\left(x_{2}(t-m h)\right),
$$

where $k_{1}>0$ and $k_{2}>0$ are the control gains, $\nu \in(-0.5,0) \cup(0,+\infty)$ is a tuning parameter. In the delay-free case with $m=0$, and without perturbations $Q_{1}$ and $Q_{2}$, this control makes the closed-loop system $\mathbf{r}$-homogeneous of degree $\nu$ with the weights $\mathbf{r}=[1,1+\nu]^{\top}$ (then we select $|x|_{r}=\left|x_{1}\right|+\left|x_{2}\right|^{\frac{1}{1+\nu}}$ ), and the system is globally finite-time stable for $\nu \in(-0.5,0)$ or globally nearly fixed-time stable for $\nu>0$. Appearance of the delay $m>0$ models the information measurement and processing lags in the control feedback. Obviously,

$$
F(\phi, \psi)=\left[\begin{array}{c}
\phi_{2} \\
-k_{1}\left|\psi_{1}\right|^{1+2 \nu} \operatorname{sign}\left(\psi_{1}\right)-k_{2}\left|\psi_{2}\right|^{\frac{1+2 \nu}{1+\nu}} \operatorname{sign}\left(\psi_{2}\right)
\end{array}\right],
$$

the assumptions 1 and 2 are satisfied (the control is continuous for $\nu>-0.5$ ), and discretization of this controlled dynamics leads to the system of interest (7).

For simulations let

$$
\begin{gathered}
k_{1}=1, k_{2}=2, h=0.01, m=10, \\
\sigma=\left\{\begin{array}{ll}
2 \nu & \nu>0 \\
\frac{\nu-r_{\min }}{2} & \nu<0
\end{array},\right. \\
Q_{1}\left(k, x^{(k)}\right)=c \sin (k h) \sum_{p=0}^{m}|x(k-p)|_{r}^{\sigma+r_{1}}, Q_{2}\left(k, x^{(k)}\right)=c \cos (2 k h) \sum_{p=0}^{m}|x(k-p)|_{r}^{\sigma+r_{2}},
\end{gathered}
$$

then for such a choice of $Q_{1}$ and $Q_{2}$ Assumption 3 is valid. Thus, all conditions of theorems 3,4 are verified. For $\nu=-0.2$ and $c=0.1$ the behavior of the norm $|x(k)|_{r}$ for three different initial conditions (they are assumed to be constant for negative values of the time) is presented in Fig. 1 in logarithmic scale. For $\nu=0.2$ and $c=10^{-2}$ the respective behavior of $|x(k)|_{r}$ is given in Fig. 2. As we can see from these plots, the convergence of the trajectories is faster than exponential (finite-time) in Fig. 1 and slower in Fig. 2, which confirms the results of corollaries 1,2 .

To apply our results it is enough to check assumptions 1 and 2, which are essential. Indeed, if we drop Assumption 1 by considering in the above system

$$
u(t)=-k_{1}\left|x_{1}(t-m h)\right|^{1+2 \nu} \operatorname{sign}\left(x_{1}(t-m h)\right)-k_{2}\left|x_{2}(t-m h)\right|^{\frac{1+2 \nu}{1+3 \nu}} \operatorname{sign}\left(x_{2}(t-m h)\right),
$$

where the power for the variable $x_{2}$ was slightly modified, $\nu>-\frac{1}{3}$, then the closed loop dynamics is still globally asymptotically stable with $m=0$ and $Q_{1}(\cdot)=Q_{2}(\cdot)=0$ (to verify this claim, a Lyapunov function 


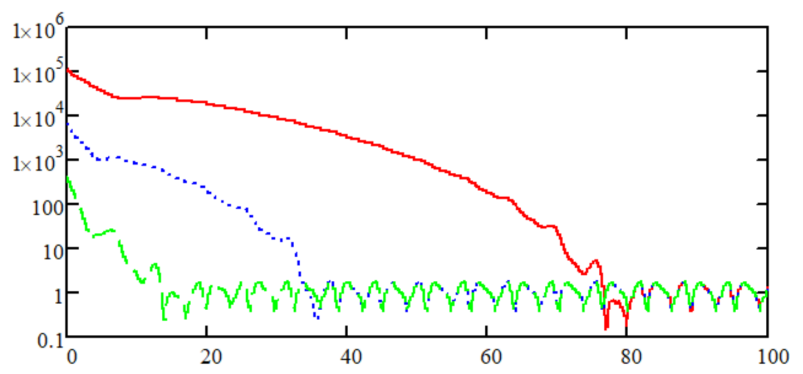

Figure 1: Trajectories of $|x(k)|_{r}$ for $\nu<0$

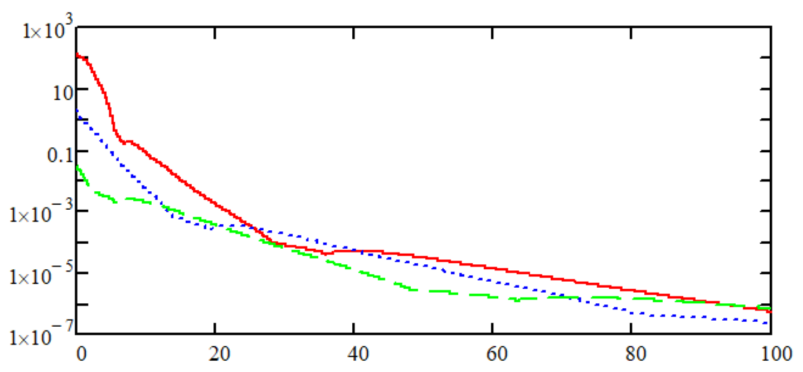

Figure 2: Trajectories of $|x(k)|_{r}$ for $\nu>0$

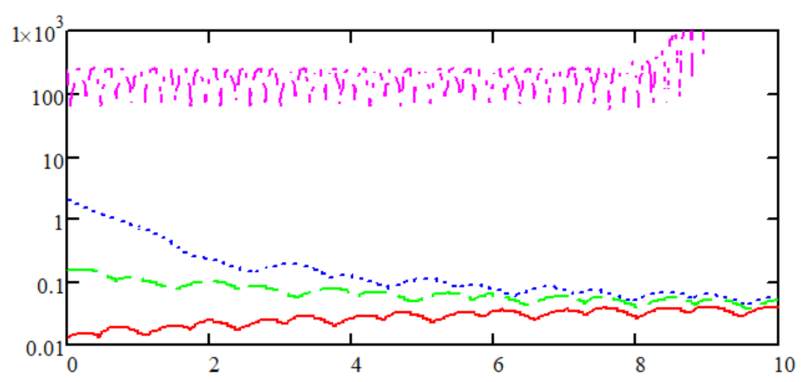

Figure 3: Trajectories of $|x(k)|_{r}$ for non-homogeneous case 
$V(x)=\frac{k_{1}}{2+2 \nu}\left|x_{1}\right|^{2+2 \nu}+\frac{1}{2} x_{2}^{2}$ can be utilized together with the LaSalle invariance principle). However, for $\nu=-0.2$ and the rest values of parameters being unchanged, the system (7) admits a locally asymptotically stable oscillating trajectory with unbounded solutions for sufficiently large initial conditions, see Fig. 3. Similar unstable behavior can be obtained by considering a homogeneous system with $\nu=0$ (the scenario not studied in this work) and a big enough discretization step $h$, since it is well-known that for linear systems the explicit Euler discretization method can be applied for a sufficiently small $h$ only.

\section{Conclusion}

The problem of stability analysis for a discrete-time time-delay system, which is derived from Euler discretization of homogeneous continuous-time model, was studied by applying the Lyapunov-Krasovskii theory. A generic structure of the Lyapunov-Krasovskii functional was proposed that can be utilized for any homogeneous system of non-zero degree. An extension of this results to the class of dynamics admitting a homogeneous approximation was developed. The obtained stability conditions were illustrated by simulation of a discretized delayed locally homogeneous planar system of negative and positive degrees. The presented results demonstrate that the explicit Euler method preserves stability properties for the selected class of homogeneous systems with non-zero degree for any value of the discretization step. Extension of this approach to the case of infinite dimensional systems with $m \rightarrow+\infty$ can be a direction of future research.

\section{References}

[1] K. Gu, V. Kharitonov, and J. Chen, Stability of Time-Delay Systems. Control Engineering, Boston: Birkhäuser, 2003.

[2] J. Hale, Theory of Functional Differential Equations. Springer-Verlag, 1977.

[3] V. Kolmanovsky and V. Nosov, Stability of functional differential equations. San Diego: CA: Academic, 1986.

[4] I. Karafyllis, M. Malisoff, F. Mazenc, and P. Pepe, eds., Recent Results on Nonlinear Delay Control Systems, vol. 4 of Advances in Delays and Dynamics. Springer International Publishing, 2016.

[5] I. Karafyllis and M. Krstic, Predictor Feedback forDelay Systems: Implementations and Approximations, ch. Discrete-time systems, pp. 251-278. Springer, 2017.

[6] L. Hetel, C. Fiter, H. Omran, A. Seuret, E. Fridman, J.-P. Richard, and S. I. Niculescu, "Recent developments on the stability of systems with aperiodic sampling: An overview," Automatica, vol. 76, pp. 309-335, 2017.

[7] J.-P. Richard, "Time-delay systems: an overview of some recent advances and open problems," Automatica, vol. 39, pp. 1667-1694, 2003.

[8] S. Monaco and D. Normand-Cyrot, "Nonlinear average passivity and stabilizingcontrollers in discrete-time," Systems $\&$ Control Letters, vol. 60, pp. 431-439, 2011.

[9] R. H. Gielen, M. Lazar, and I. V. Kolmanovsky, "Lyapunov methods for time-invariant delay difference inclusions," SIAM Journal on Control and Optimization, vol. 50, no. 1, pp. 110-132, 2012.

[10] E. Fridman, Introduction to Time-Delay Systems: Analysis and Control. Basel: Birkhäuser, 2014.

[11] J. Schiffer, E. Fridman, R. Ortega, and J. Raisch, "Stability of a class of delayed port-Hamiltonian systems with application to microgrids with distributed rotational and electronic generation," Automatica, vol. 74, pp. 71-79, 2016.

[12] E. Bernuau, D. Efimov, W. Perruquetti, and A. Polyakov, "On homogeneity and its application in sliding mode," Journal of the Franklin Institute, vol. 351, no. 4, pp. 1866-1901, 2014.

[13] A. Bacciotti and L. Rosier, Liapunov Functions and Stability in Control Theory, vol. 267 of Lecture Notes in Control and Inform. Sci. Berlin: Springer, 2001.

[14] S. Bhat and D. Bernstein, "Geometric homogeneity with applications to finite-time stability," Mathematics of Control, Signals and Systems, vol. 17, pp. 101-127, 2005.

[15] M. Kawski, Homogeneous feedback stabilization, vol. 7 of Progress in systems and control theory: New trends in systems theory. Birkhäuser, 1991. 
[16] V. Zubov, "On systems of ordinary differential equations with generalized homogenous right-hand sides," Izvestia vuzov. Mathematica., vol. 1, pp. 80-88, 1958. in Russian.

[17] A. Filippov, "Stability of differential equations with discontinuous and multi-valued right-hand parts," Differential equations, vol. 15, no. 6, pp. 1018-1027, 1979.

[18] A. Levant, "Homogeneity approach to high-order sliding mode design," Automatica, vol. 41, no. 5, pp. 823$830,2005$.

[19] D. Efimov, W. Perruquetti, and J.-P. Richard, "Development of homogeneity concept for time-delay systems," SIAM J. Control Optim., vol. 52, no. 3, pp. 1403-1808, 2014.

[20] D. Efimov, A. Polyakov, W. Perruquetti, and J.-P. Richard, "Weighted homogeneity for time-delay systems: Finite-time and independent of delay stability," IEEE Trans. Automatic Control, vol. 61, no. 1, pp. 210-215, 2016.

[21] T. Sanchez, D. Efimov, A. Polyakov, J. Moreno, and W. Perruquetti, "On homogeneity of discrete-time systems: stability and convergence rates," Int. J. Robust and Nonlinear Control, vol. 29, no. 8, pp. 2406$2421,2019$.

[22] A. Polyakov, D. Efimov, E. Fridman, and W. Perruquetti, "On homogeneous distributed parameter systems," IEEE Trans. Automatic Control, vol. 61, no. 11, pp. 3657-3662, 2016.

[23] A. Kanevskiy and L. Reyzign, "Design of homogeneous Lyapunov-Krasovskii functions," Differential equations, vol. 9, no. 2, pp. 251-259, 1973.

[24] L. Rosier, "Homogeneous Lyapunov function for homogeneous continuous vector field," Systems\&Control Lett., vol. 19, pp. 467-473, 1992.

[25] D. Efimov and W. Perruquetti, "Oscillations conditions in homogenous systems," in Proc. NOLCOS'10, (Bologna), pp. 1379-1384, 2010.

[26] D. Efimov, W. Perruquetti, and M. Petreczky, "On necessary conditions of instability and design of destabilizing controls," in Proc. 53rd IEEE CDC, (LA), 2014.

[27] D. Efimov, R. Ushirobira, J. Moreno, and W. Perruquetti, "Homogeneous lyapunov functions: from converse design to numerical implementation," SIAM J. Control Optimization, vol. 56, no. 5, pp. 3454-3477, 2018.

[28] E. Bernuau, A. Polyakov, D. Efimov, and W. Perruquetti, "Verification of iss, iiss and ioss properties applying weighted homogeneity," Systems 6 Control Letters, vol. 62, no. 12, pp. 1159-1167, 2013.

[29] A. Aleksandrov and A. Zhabko, "Delay-independent stability of homogeneous systems," Applied Mathematics Letters, vol. 34, no. 8, pp. 43-50, 2014.

[30] K. Zimenko, D. Efimov, A. Polyakov, and W. Perruquetti, "A note on delay robustness for homogeneous systems with negative degree.," Automatica, vol. 79, no. 5, pp. 178-184, 2017.

[31] V. Andrieu, L. Praly, and A. Astolfi, "Homogeneous approximation, recursive observer design, and output feedback," SIAM J. Control Optimization, vol. 47, no. 4, pp. 1814-1850, 2008.

[32] D. Efimov and W. Perruquetti, "Conditions of oscillations and multi-homogeneity," Mathematics of Control, Signals, and Systems, vol. 28, no. 3, pp. 1-37, 2016.

[33] J. Diblik, "Asymptotic equilibrium for homogeneous delay linear differential equations with l-perturbation term," Nonlinear Analysis, Theory, Methods 6 Applicarions, vol. 30, no. 6, pp. 3927-3933, 1997.

[34] F. Asl and A. Ulsoy, "Analytical solution of a system of homogeneous delay differential equations via the Lambert function," in Proc. American Control Conference, (Chicago), pp. 2496-2500, 2000.

[35] F. Mazenc, S. Mondie, and S.-I. Niculescu, "Global asymptotic stabilization for chains of integrators with a delay in the input," in Proc. 40th IEEE Conference on Decision and Control, vol. 2, pp. 1843-1848, 2001.

[36] V. Bokharaie, O. Mason, and M. Verwoerd, "D-stability and delay-independent stability of homogeneous cooperative systems," IEEE Trans. Automatic Control, vol. 55, no. 12, pp. 2882-2885, 2010.

[37] A. Aleksandrov and A. Zhabko, "On the asymptotic stability of solutions of nonlinear systems with delay," Siberian Mathematical Journal, vol. 53, no. 3, pp. 393-403, 2012. 
[38] A. Aleksandrov, A. Zhabko, and V. Pecherskiy, "Complete type functionals for some classes of homogeneous diffrential-difference systems," in Proc. 8th international conference "Modern methods of applied mathematics, control theory and computer technology", (Voronezh), pp. 5-8, 2015. in Russian.

[39] D. Efimov and A. Aleksandrov, "Analysis of robustness of homogeneous systems with time delays using Lyapunov-Krasovskii functionals," Int. J. Robust and Nonlinear Control, 2020.

[40] A. Aleksandrov and A. Zhabko, "On stability of solutions to one class of nonlinear difference systems," Siberian Math. J., vol. 44, no. 6, pp. 951-958, 2003.

[41] D. Efimov, A. Polyakov, A. Levant, and W. Perruquetti, "Realization and discretization of asymptotically stable homogeneous systems," IEEE Transactions on Automatic Control, vol. 62, no. 11, pp. 5962-5969, 2017.

[42] V. Kharitonov and A. Zhabko, "Lyapunov-Krasovskii approach to the robust stability analysis of time-delay systems," Automatica, vol. 39, no. 1, pp. 15-20, 2003. 